\title{
FUNDAMENTAL LIMITATIONS OF POLYNOMIAL CHAOS FOR UNCERTAINTY QUANTIFICATION IN SYSTEMS WITH INTERMITTENT INSTABILITIES*
}

\author{
MICHAL BRANICKI ${ }^{\dagger}$ AND ANDREW J. MAJDA ${ }^{\ddagger}$
}

\begin{abstract}
Here, we examine the suitability of truncated Polynomial Chaos Expansions (PCE) and truncated Gram-Charlier Expansions (GrChE) as possible methods for uncertainty quantification (UQ) in nonlinear systems with intermittency and positive Lyapunov exponents. These two methods rely on truncated Galerkin projections of either the system variables in a fixed polynomial basis spanning the 'uncertain' subspace (PCE) or a suitable eigenfunction expansion of the joint probability distribution associated with the uncertain evolution of the system ( $\mathrm{GrChE})$. Based on a simple, statistically exactly solvable non-linear and non-Gaussian test model, we show in detail that methods exploiting truncated spectral expansions, be it PCE or GrChE, have significant limitations for uncertainty quantification in systems with intermittent instabilities or parametric uncertainties in the damping. Intermittency and fat-tailed probability densities are hallmark features of the inertial and dissipation ranges of turbulence and we show that in such important dynamical regimes PCE performs, at best, similarly to the vastly simpler Gaussian moment closure technique utilized earlier by the authors in a different context for UQ within a framework of Empirical Information Theory. Moreover, we show that the non-realizability of the GrChE approximations is linked to the onset of intermittency in the dynamics and it is frequently accompanied by an erroneous blow-up of the second-order statistics at short times. These limitations of the two types of truncated spectral expansions arise from the following: (i) Non-uniform convergence in time of PCE and GrChE resulting in a rapidly increasing number of terms necessary for a good approximation of the random process as time evolves, (ii) Fundamental problems with capturing the constant flux of randomness due to white Gaussian noise forcing via finite truncations of the spectral representation of the associated Wiener process, (iii) Slow decay of PCE and GrChE coefficients in the presence of intermittency, hampering implementation of sparse truncation methods which have been widely used in nearly elliptic problems or in low Reynolds number flows. Rigorous justification of these limitations is richly illustrated by straightforward tests exploiting a simple nonlinear and non-Gaussian but statistically exactly solvable test model which is proposed here as a challenging benchmark for algorithms for UQ in systems with intermittency.
\end{abstract}

Key words. Polynomial Chaos, uncertainty quantification, intermittency, white noise, parametric uncertainty.

AMS subject classifications. 60H10, 60H30, 35C20.

\section{Introduction}

The theory of homogeneous chaos of Wiener [38], proposed initially for use in the statistical theory of turbulence [39] and widely discarded some thirty years later (e.g. $[34,5,6])$, has recently regained its popularity as a method for quantifying propagation of uncertainty in nonlinear dynamical systems. Uncertainty Quantification (UQ) is undoubtedly an important issue in many physics, engineering, and geoscience applications where complex nonlinear interactions of resolved and unresolved processes and/or parametric uncertainty need to be properly treated in order to estimate the uncertain evolution of the usually high-dimensional system. Practical implementations of the Wiener Chaos framework for UQ, commonly referred to as Polynomial Chaos Expansions (PCE), rely on a truncated spectral expansion of the system variables via Jin.

${ }^{*}$ Received: January 18, 2012; accepted(in revised form): April 15, 2012. Communicated by Shi

${ }^{\dagger}$ Department of Mathematics and Center for Atmosphere Ocean Science, Courant Institute of Mathematical Sciences, New York University, New York, USA (branicki@cims.nyu.edu).

${ }^{\ddagger}$ Department of Mathematics and Center for Atmosphere Ocean Science, Courant Institute of Mathematical Sciences, New York University, New York, USA. 
a (truncated) Galerkin-type projection onto a space spanned by a fixed orthonormal polynomial basis in random variables (see, e.g., $[11,40,41,42,43,13,24]$ for but a few examples). In most applications such UQ techniques are restricted to estimating the second-order statistics of the resolved variables, i.e., means and variances. The PCE framework has many attractive features which are potentially well suited for numerical computations with often a substantially smaller computational effort compared to Monte Carlo sampling. These include a desirable separation of the random and deterministic components of the dynamics and 'realizability' of the resulting approximations, i.e., the fact that the expected values of various dynamical quantities can be realized as averages with respect to a nonnegative probability density. The realizability property is particularly useful since it is well known that truncated moment expansions of the associated probability densities, i.e., the Gram-Charlier expansions ( $\mathrm{GrChE})$, can lead to non-realizability and negative energy spectra [32, 34, 5].

However, despite the well documented success stories which are predominantly confined to UQ at short times in systems with parametric uncertainty or UQ in elliptic or low Reynolds number problems in fluid dynamics (e.g., [13, 35, 24, 8]), there are many well known remaining limitations of the PCE approach. These include UQ in deterministic systems with parametric uncertainty and nontrivial attractors (i.e., limit cycles, strange attractors, etc. [29]), or long time integration of systems driven by white noise (e.g., $[12,19,20])$. The latter limitations are particularly important for UQ in high-dimensional nonlinear turbulent systems where small scale unresolved processes modeled as idealized white Gaussian noise interact nontrivially with the resolved processes. Similarly, many of the small scale effects and various uncertainties in other applications and areas, including physics, material science, chemistry biology, etc., can be naturally modeled by a white noise process with the resolved processes/fields satisfying appropriate Stochastic Differential Equations (SDEs, e.g., [33]) or Stochastic Partial Differential Equations (SPDEs, e.g., [30]).

In this paper we utilize a simple unambiguous test model consisting of two coupled SDEs introduced below and show that methods exploiting truncated spectral expansions, be it $\mathrm{PCE}$ or $\mathrm{GrChE}$, have additional severe limitations for UQ in systems with intermittency or parametric uncertainty in the damping and fat-tailed probability densities, even in the absence of explicit time dependence, or nontrivial attractors. Intermittency and fat-tailed probability densities are abundant in the inertial and dissipation range of stochastic turbulence models (e.g., [7]) and we show that in such important dynamical regimes PCE performs, at best, similarly to the simple Gaussian moment closure technique utilized earlier in a different context for UQ within a framework of Empirical Information Theory [3]. Moreover, we show that the nonrealizability of the $\mathrm{GrChE}$ approximations is linked to the onset of intermittency in the dynamics and it is frequently accompanied by an erroneous blow-up of the secondorder statistics at short times. These limitations presented here in an unambiguous test model are similar to those encountered in heuristic applications to turbulence theory and stem mainly from the following:

- The finite truncation of the spectral expansions of either the solution process (PCE), or of the probability density ( $\mathrm{GrChE})$.

- Non-uniform convergence in time of PCE which leads to a rapidly increasing number of terms that are necessary to get a good approximate representation of the random process as time evolves.

- Constant flux of randomness due to white noise forcing and fundamental problems with capturing this flux via finite truncations of the spectral repre- 
sentation of the associated Wiener process.

- Slow decay of the PCE and GrChE coefficients in the presence of intermittency which hampers implementation of existing sparse truncation methods widely used in elliptic problems or in low Reynolds number flows (e.g., $[13,35,24,8])$.

Applications of the PCE framework to stochastic PDEs considered in the literature are predominantly concerned with UQ in systems with a finite (and usually small) number of random variables with known distributions (see, e.g., [31, 11, 40, 41, 42, $43,44,45,36]$ ). Over the past decade the efforts to improve the PCE approximation of stochastic dynamics with such 'low-dimensional' parametric uncertainty have led to the development of various techniques aimed at mitigating the shortcomings associated with the finite truncations of the spectral expansions in the presence of explicit time dependence and nontrivial attractors. A common problem in such cases, which also occurs in the case of white noise forcing discussed below, is that the number of terms required for maintaining the desired accuracy of the solution grows rapidly with the integration time. In this context a number of techniques based either on expansion over piecewise (local) polynomial bases [15, 16, 36, 37], or multi-resolution schemes $[17,28]$ were developed. However, while often useful, these techniques merely reduce the computational overhead by dynamically adapting the PCE basis in an effort to maintain a small number of expansion coefficients, which nevertheless continues to grow and eventually becomes very large [23]; such techniques also quickly lose efficiency when the number of uncertain parameters increases [36]. Other methods, relying on the so-called asynchronous time integration [21, 1] introduce suitable transformations of the model variables in such a way that a low-order truncated PCE basis remains suitable for arbitrarily large times. However, these techniques seem inherently designed for UQ in systems with parametric uncertainty and require existence of a reference deterministic system with a structurally stable attractor with no positive Lyapunov exponents so that the trajectories on the attractors of surrogate systems with different realizations of the uncertain parameters remain 'close' to the reference trajectories.

UQ based on Polynomial Chaos Expansions in systems with white noise forcing is known to be substantially more complicated [19, 12, 20] since, in addition to the aforementioned problems, one has to deal in such cases with, effectively, an infinite number of random variables necessary for representing the white noise process. Nevertheless, accurate PCE approximations of uncertain dynamics can be achieved at short times in nearly Gaussian systems $[19,12,20]$ where sparse truncation methods can be employed in order to 'concentrate' the truncated random basis on the low-order coefficients of the spectral expansion. However, as we argue below based on simple examples, implementation of the existing sparse truncation methods (e.g., $[13,35,24,8])$ in systems with white noise driven intermittency is hampered by a slow decay of the amplitudes of the expansion coefficients. Consequently, the number of terms in the PCE approximation quickly grows in time beyond computationally acceptable limits. Incidentally, similar conclusions were made in the past [6] in the context of truncated expansions of Burgers equation where it was argued that PCE approximations cannot reproduce the energy cascade due to insufficiently rapid energy decay at $R e \gg 1$ and high order PCE terms are needed even for nearly Gaussian turbulent fields.

In order to address and illustrate the issues mentioned above in an unambiguous fashion, we consider here the simplest test model with white noise driven intermit- 
tency and quadratic nonlinearity allowing for a straightforward comparison of different techniques for uncertainty quantification. To this end, we study the following twodimensional and conditionally Gaussian (see, e.g., [18]) system of coupled Itô SDE's given by

$$
\begin{aligned}
& \text { a) } \mathrm{d} u(t)=\left(-\left(\hat{\gamma}+a_{u} \gamma(t)\right) u(t)+f(t)\right) \mathrm{d} t+\sigma_{u} \mathrm{~d} W_{u}(t), \\
& \text { b) } \mathrm{d} \gamma(t)=-\left(d_{\gamma}+a_{\gamma} u(t)\right) \gamma(t) \mathrm{d} t+\sigma_{\gamma} \mathrm{d} W_{\gamma}(t),
\end{aligned}
$$

where $u, \gamma$ are the two real variables, $\hat{\gamma}, d_{\gamma}>0$ are the damping parameters, $\sigma_{u}, \sigma_{\gamma}>0$ are the noise amplitudes, and $W_{u}, W_{\gamma}$ are two independent scalar Wiener processes. Based on the previous analysis (see $[2,9,10,3]$ and Section 3.1) it is known that even the simplified system (1.1) with $a_{\gamma}=0$ possesses various interesting dynamical regimes with very intermittent transient instabilities as long as $a_{u} \neq 0$ which lead to fat-tailed PDFs with only a small number of finite moments; such configurations are typical in realistic turbulent signals in the energy transfer or energy dissipation regimes (see figure 1.1).
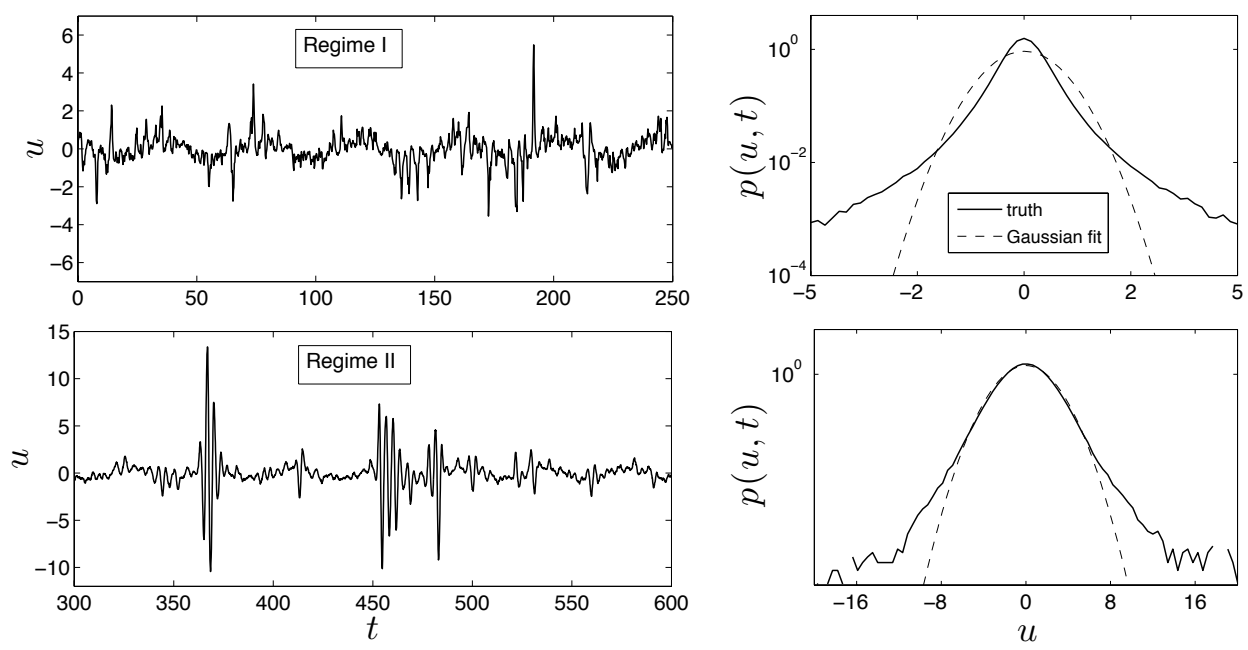

FIG. 1.1. Schematic illustration of path-wise dynamics (left) and time-periodic statistics on the attractor (right) in two intermittent regimes of system (1.1); the intermittent regimes are discussed in Section 3.

The test model (1.1) which is proposed as a challenging, yet mathematically tractable, benchmark for testing UQ techniques in the important class of systems with intermittency, has a number of attractive properties:

- Very rich dynamical behavior, including a variety of intermittent regimes with a positive Lyapunov exponent, which allows for testing various methods in a wide range of realistic complex dynamics.

- Exact statistical solvability for $a_{\gamma}=0$ in (1.1) with exact analytical formulas for the second-order statistics derived in [9].

- The time-dependent PDFs for $u$ in (1.1) can be obtained with high precision by simple numerics and in the white noise limit of rapidly decorrelating damping fluctuations $\gamma$ the exact equilibrium PDF is known [2]. 
We argue that, due to the combination of analytical tractability and very rich dynamical behavior with intermittency and a positive Lyapunov exponent, the system (1.1) represents a very attractive test model for analyzing the performance of various techniques for uncertainty quantification in turbulent dynamical systems. Consequently, we analyze below the performance of the following two approaches to approximating the statistical solutions of the system (1.1):

- Truncated Polynomial Chaos Expansions (PCE) of $u, \gamma$ in (1.1).

- Truncated eigenfunction expansion of the joint probability density $p(u, \gamma, t)$ satisfying the Fokker-Planck equation associated with (1.1), i.e. the GramCharlier Expansions (GrChE).

Various unambiguous tests are presented in the following sections, all of which point to the severe limitations of PCE and GrChE in systems with white noise driven intermittency.

This paper is structured as follows. First, in Section 2 we outline two techniques which are commonly used in various incarnations for uncertainty quantification in nonlinear dynamical systems. Both these methods rely on finite truncations of infinite functional expansions of either the solution process itself or the associated probability density function. In Section 3 we discuss the exactly solvable test model with intermittent dynamics in more detail and introduce three particular configurations in Section 3.1 which are used afterwards to analyze the performance of the PCE and GrChE methods in various dynamical configurations. The limitations of the PCE and $\mathrm{GrChE}$ techniques for uncertainty quantification in systems with white noise driven intermittency are discussed in sections 4 and 5 based on various examples of increasing complexity; more detailed explanation of the limitations of these two methods and rigorous derivations are given in extensive appendices referenced throughout the paper. Finally, we present some concluding remarks and outline challenges for further research in this area in Section 6.

2. Polynomial Chaos Expansions (PCE), Gram-Charlier Expansions ( $\mathrm{GrChE})$, and their finite truncations

Here, we briefly recapitulate basic properties of two different methods for uncertainty quantification in systems evolving subject to nonlinear interactions with unresolved processes and parametric uncertainty. The first technique, the Polynomial Chaos Expansion (PCE), relies on approximating the uncertain state of the system satisfying the appropriate Stochastic Differential Equation (SDE; e.g., [33]). The second approach, outlined in Section 2.2, is based on the Gram-Charlier expansions of the associated probability density function which satisfies the appropriate FokkerPlanck equation. More details concerning these two techniques for UQ are discussed in Appendices A and B.

As already mentioned in the introduction, we are mainly concerned here with uncertainty quantification in turbulent nonlinear systems with intermittency and positive Lyapunov exponents where nontrivial interactions with unresolved processes are important for the mean dynamics. Below we show that representation of these unresolved processes as idealized white noise drastically complicates the problem of uncertainty quantification in such systems via the PC or GrCh expansions, since the finite truncations of these expansions struggle with capturing the constant influx of new randomness and its 'redistribution' through the nonlinear interactions. Limitations of these techniques for UQ in systems with intermittency are discussed in Section 4 and Section 5. 
2.1. Finite truncation of a Polynomial Chaos Expansion of a stochastic process. Polynomial Chaos Expansion (PCE) is unique among the existing approaches to uncertainty quantification as it deals directly with the random process/field representing the uncertain evolution of the system in question rather than with moments of the associated probability density function. The PCE of a solution of an SDE (e.g., [33]) or SPDE (e.g., [30]) separates the deterministic effects from the randomness arising due to the nonlinear interactions with unresolved processes. An expansion of the system variables is made via a Galerkin-type projection of the model variables onto the space spanned by the fixed orthonormal polynomial basis in random variables. In the case of the so-called Wiener-Hermite expansion, the first non-constant term in the expansion is Gaussian. It is well known (see [4] and Appendix A) that the exact infinite expansion converges but it involves an infinite number of coefficients; the convergence is, however, not uniform in time and the expansion coefficients are related by an infinite-dimensional set of ODEs in an infinite number of unknowns. The need for finite truncations of these expansions required in applications leads to important limitations for UQ in systems with intermittency, as discussed in Section 4.

The main properties of the PCE framework are as follows: Denote the set of multi-indices with finite number of nonzero components as

$$
\mathcal{J}=\left\{\alpha=\left(\alpha_{i}, i \geqslant 1 \mid \alpha_{i} \in\{0,1,2, \ldots\}\right),|\alpha|=\sum_{i=1}^{\infty} \alpha_{i}<\infty\right\} .
$$

For an index $\alpha=\left(\alpha_{1}, \alpha_{2}, \ldots\right) \in \mathcal{J}$ a multivariate Hermite polynomial in an infinite number of i.i.d. Gaussian random variables $\boldsymbol{\xi}=\left(\xi_{1}, \xi_{2}, \ldots\right),\left\langle\xi_{i}\right\rangle=0,\left\langle\xi_{i} \xi_{j}\right\rangle=\delta_{i j}$, is

$$
T_{\alpha}(\boldsymbol{\xi})=\prod_{i=1}^{\infty} H_{\alpha_{i}}\left(\xi_{i}\right)
$$

where $H_{n}(x)$ is the normalized $n$-th order Hermite polynomial. For $\alpha \in \mathcal{J}$, the product in the right hand side of (2.2) has a finite number of factors and it is well defined. The random functions $T_{\alpha}$ are called Wick polynomials and they form a complete orthonormal basis in $L^{2}$ on the probability space with respect to the Gaussian measure generated by $\xi$ so that

$$
\left\langle T_{\alpha} T_{\beta}\right\rangle=\delta_{\alpha \beta}, \quad\left\langle T_{0}\right\rangle=1, \quad\left\langle T_{\alpha}\right\rangle=0 \text { when } \alpha \neq(0,0, \ldots) .
$$

The order of the Wick polynomial $T_{\alpha}$ is defined as $|\alpha|=\sum \alpha_{i}$ and $\alpha=\beta$ when $\alpha_{i}=\beta_{i}$ for all $i$.

According to the Cameron-Martin Theorem [4], any process such that $\left\langle|u(t, \boldsymbol{\xi})|^{2}\right\rangle<\infty$ has the following expansion in terms of the orthonormal Wick polynomials $T_{\alpha}$ (see Appendix A for more details),

$$
u(t, \boldsymbol{\xi})=\sum_{\alpha \in \mathcal{J}} u_{\alpha}(t) T_{\alpha}(\boldsymbol{\xi}), \quad u_{\alpha}(t)=\left\langle u(t, \boldsymbol{\xi}) T_{\alpha}(\boldsymbol{\xi})\right\rangle .
$$

Furthermore, the first two statistical moments of $u(x, s, \boldsymbol{\xi})$ are given by:

$$
\langle u(t, \boldsymbol{\xi})\rangle=u_{0}(t), \quad\left\langle|u(t, \boldsymbol{\xi})|^{2}\right\rangle=\sum_{\alpha \in \mathcal{J}}\left|u_{\alpha}(t)\right|^{2} .
$$


The expansion (2.4) is often called the Polynomial Chaos Expansion (PCE) or Wiener-Hermite Chaos Expansion when the Hermite polynomials are used in (2.2); a more general form of the expansion $(2.4)$ for a random field $u(x, t, \boldsymbol{\xi})$ is given in Appendix A.

The doubly infinite expansion (2.4) is useless in applications unless a suitable truncation is employed. The simplest such truncation is obtained by defining the truncated index set

$$
\mathcal{J}_{K, N}=\left\{\alpha=\left(\alpha_{i}, i \geqslant 1 \mid \alpha_{i} \in\{0,1,2, \ldots, K\}\right),|\alpha|=\sum_{i=1}^{K} \alpha_{i} \leqslant N\right\},
$$

accounting for only the first $K$ random Gaussian variables $\xi_{i}$ and the Wick polynomials up to order $N$. Then, the truncated PCE of $u(t, \boldsymbol{\xi})$ has the form

$$
u_{K, N}(t, \boldsymbol{\xi})=\sum_{\alpha \in \mathcal{J}_{K, N}} u_{\alpha}(t) T_{\alpha}(\boldsymbol{\xi})=\sum_{|\alpha| \leqslant N} u_{\alpha}(t) \prod_{i=1}^{K} H_{\alpha_{i}}\left(\xi_{i}\right),
$$

so that the resulting approximation has altogether $\sum_{n=0}^{N}\left(\begin{array}{c}K+n-1 \\ n\end{array}\right)$ terms; clearly the number of coefficients in the truncated expansion grows rapidly with increasing $K$ and/or $N$. In order to reduce the number of expansion coefficients and the computational overhead, Luo [19, 20] and Hou et al. [12] proposed a sparse truncation method which relied on (i) the use of lower order polynomials for $\xi_{i}$ with higher subscripts and (ii) decoupling the random variables with higher subscripts from the random variables with lower subscripts (the threshold between 'lower' and 'higher' subscripts depended on applications). Similar sparse truncation methods were proposed for elliptic problems in $[35,8]$. We will show in Section 4 that truncation methods of this type are not adequate for turbulent systems with energy transfer on the attractor and/or intermittency due to a slow decay of amplitudes of the expansion coefficients. In such systems neglecting higher order terms in the expansions of nonlinear terms has particularly severe consequences for the accuracy of the truncated approximations even without the sparse truncation.

2.1.1. Truncated equations for the PCE coefficients of the test model with intermittency. The test model (1.1) has no spatial dependence, which simplifies the expansion (2.4) and its finite truncation (2.7) for the system variables $u$ and $\gamma$. However, since we are concerned here with uncertainty quantification in systems driven in part by Brownian motion due to nonlinear interactions with unresolved processes, it is necessary to incorporate into the PCE framework the effects of constant flux of new randomness due to the white noise forcing. We follow here the approach of $[19,20]$ and $[12]$ and consider an orthonormal basis $\left\{m_{i}(s), i=1,2, \ldots\right\}$ in $L^{2}([0, T])$ with $T>0$, e.g., trigonometric functions (see Appendix A), to construct a spectral representation of the Wiener process $W(t)$ in the following way:

Given the infinite set of i.i.d. Gaussian random variables $\boldsymbol{\xi}=\left\{\xi_{i}\right\}_{i=1,2, \ldots, \infty}$, $\xi_{i} \sim \mathcal{N}(0,1)$, where

$$
\xi_{i}=\int_{0}^{T} m_{i}(s) \mathrm{d} W(s)
$$

the Wiener process can be represented in the Fourier-Hermite series as

$$
W(s)=\sum_{i=1}^{\infty} \xi_{i} \int_{0}^{s} m_{i}(\tau) \mathrm{d} W(\tau) .
$$


We assume here without a proof that solutions of (1.1) are second-order stable (methods similar to those used in [2] could be used to prove the second-order stability of (1.1) when $a_{\gamma}=0$; otherwise, careful Monte Carlo estimates are sufficient for our purposes). By the Cameron-Martin Theorem (see [4] and Appendix A) these solutions admit the PCE expansion (2.4) so that the truncated equations for the PCE coefficients with $\alpha \in \mathcal{J}_{K, N}$ of the system (1.1) are

$$
\begin{aligned}
& \text { a) } \dot{u}_{\alpha}=-\hat{\gamma} u_{\alpha}-a_{u} \sum_{p \in \mathcal{J}_{K, N}} \sum_{0 \leqslant \beta \leqslant \alpha} C(\alpha, \beta, p) u_{\alpha-\beta+p} \gamma_{\beta+p}+f(t) \delta_{\alpha \mathbf{0}}+\sigma_{u} \sum_{i \in \mathcal{K}_{u}} I_{\left\{\alpha_{j}=\delta_{i j}\right\}} m_{i}(t) \text {, } \\
& \text { b) } \dot{\gamma}_{\alpha}=-d_{\gamma} \gamma_{\alpha}-a_{\gamma} \sum_{p \in \mathcal{J}_{K, N}} \sum_{0 \leqslant \beta \leqslant \alpha} C(\alpha, \beta, p) u_{\alpha-\beta+p} \gamma_{\beta+p}+\sigma_{\gamma} \sum_{i \in \mathcal{K}_{\gamma}} I_{\left\{\alpha_{j}=\delta_{i j}\right\}} m_{i}(t) \text {, }
\end{aligned}
$$

where $\mathcal{K}_{u}, \mathcal{K}_{\gamma}$ are two sets of integers which are disjoint if the white noises $\dot{W}_{u}, \dot{W}_{\gamma}$ are independent, the Kronecker delta $\delta_{\alpha \mathbf{0}} \neq 0$ only if $\alpha=(0,0, \ldots)$, and the indicator function, $I_{\alpha_{j}=\delta_{i j}}$, equals 1 if $\alpha_{j}=\delta_{i j}$ and equals zero otherwise. In deriving (2.10), which is discussed in more detail in Appendix A.3, we utilized the orthonormality of the Wick polynomials $T_{\alpha}$ together with the following important identity which allows for expressing the nonlinear terms in (1.1) via infinite expansions in the Wick polynomials (see $[27,19,12,20]$ and Theorem 2 of Appendix A):

$$
u \gamma=\sum_{\alpha \in \mathcal{J}}\left(\sum_{p \in \mathcal{J}} \sum_{0 \leqslant \beta \leqslant \alpha} C(\alpha, \beta, p) u_{\alpha-\beta+p} \gamma_{\beta+p}\right) T_{\alpha} .
$$

The white-noise forcing in the above truncated deterministic system of ODEs for the coefficients $u_{\alpha}, \gamma_{\alpha}$ is represented by the $K$ basis functions $m_{i}(t)$. Note that the white noise forcing does not directly affect the evolution of the mean, which is given by $u_{0}(t)$ by the Cameron-Martin Theorem (Appendix A), but the presence of nonlinearities in (2.10) results in the backscatter of the stochastic noise into the mean dynamics. Note that truncating (2.11) results in neglecting, at least partially, the effects of small scale fluxes on the 'resolved' dynamics $u(t)$ which is particularly important in the presence of intermittency and non-vanishing energy transfer between Fourier modes of $u$ at equilibrium.

Limitations of the truncated PCE method for solving white noise driven dynamics were noted in [12] where reliable integration of the uncertain dynamics required an unreasonably large dimension of the random vector $\boldsymbol{\xi}$, except for sufficiently short times. We will show in Section 4 that the performance of this method is diminished further for UQ in systems with intermittency driven by unresolved (white noise) processes, or even in systems with parametric uncertainty associated with fat-tailed probability densities, where finite truncations of (2.9) combined with neglecting higher order terms in the expansion (2.11) of nonlinearities in (1.1) have severe consequences on the estimated statistics of the solutions.

2.2. Gram-Charlier Expansions (GrChE) of the probability density. We outline here another method for estimating the uncertain evolution of a system modeled as a diffusion process (i.e., satisfying an Itô SDE) by expanding the associated probability density in orthonormal eigenfunctions of the Fokker-Planck equation. We illustrate the necessary steps of this procedure for UQ on the test system (1.1) where the appropriate eigenfunction set turns out to be the parabolic cylinder functions and the second-order statistics depend only on three low order coefficients in the infinite 
expansion of the probability density; details of the derivations below are presented in Appendix B.

Some limitations of the Gram-Charlier expansion have already been known from applications in turbulence theory $[32,5]$. While practical implementation of this method does not require any finite spectral truncation of the the Wiener process as in the PCE method (cf. Section 2.1.1), we will show in Section 5 that it too possesses a number of important limitations for UQ in systems with intermittency such as nonrealizability of the PDFs and even erroneous blow-up of low order statistics at short times.

The main steps of the GrChE method are as follows. Consider the Fokker-Planck equation associated with (1.1), which is given by

$$
\partial_{t} p(u, \gamma, t)=\mathcal{D}_{u} \partial_{u}^{2} p+\hat{\gamma} \partial_{u}[u p]+\mathcal{D}_{\gamma} \partial_{\gamma}^{2} p+d_{\gamma} \partial_{\gamma}[\gamma p]+\partial_{u}\left[\left(a_{u} \gamma u-f\right) p\right]+a_{\gamma} u \partial_{\gamma}[\gamma p],
$$

where $\mathcal{D}_{u}=\sigma_{u}^{2} / 2, \mathcal{D}_{\gamma}=\sigma_{\gamma}^{2} / 2$. Equation (2.12) can be written as

$$
\partial_{t} p(u, \gamma, t)=\mathcal{L}_{F P}^{u} p+\mathcal{L}_{F P}^{\gamma} p+\partial_{u}\left[\left(a_{u} \gamma u-f\right) p\right]+a_{\gamma} u \partial_{\gamma}[\gamma p],
$$

where the first two terms

$$
\mathcal{L}_{F P}^{u} p=\mathcal{D}_{u} \partial_{u}^{2} p+\hat{\gamma} \partial_{u}[u p], \quad \mathcal{L}_{F P}^{\gamma} p=\mathcal{D}_{\gamma} \partial_{\gamma}^{2} p+d_{\gamma} \partial_{\gamma}[\gamma p],
$$

are the Fokker-Planck operators associated with uncoupled OU processes involving either $u$ or $\gamma$, and the last two terms in (2.13) represent the quadratic coupling between the two processes. In the approximation procedure we first consider the Fokker-Planck equation without the quadratic coupling terms, i.e.,

$$
\partial_{t} p(u, \gamma, t)=\mathcal{L}_{F P}^{u} p+\mathcal{L}_{F P}^{\gamma} p
$$

and observe (see the Appendix B) that (2.15) is solved exactly by

$$
p(u, \gamma, t)=\sum_{k, l=0}^{\infty} C_{k, l} \phi_{k}(u) \phi_{l}(\gamma),
$$

where the orthonormal basis functions $\phi_{k}(u) \phi_{\beta}(\gamma)$ are a product of eigenfunctions of the operators $\mathcal{L}_{F P}^{u}$ and $\mathcal{L}_{F P}^{\gamma}$ respectively, and the time-dependent coefficients $C_{k, l}$ satisfy an infinite-dimensional system of ODEs.

Any practical application of the expansion (2.16) requires a finite-dimensional truncation which leads to

$$
p^{\boldsymbol{M}}(u, \gamma, t)=\sum_{\substack{0 \leqslant k \leqslant K, 0 \leqslant l \leqslant L}} C_{k, l}^{\boldsymbol{M}} \phi_{k}(u) \phi_{l}(\gamma), \quad \boldsymbol{M}=(K, L),
$$

where the coefficients $C_{k, l}^{M}$ in the truncated expansion (2.17) satisfy a system of linearly coupled ODEs (see Appendix B and equation (B.36) for details)

$$
\frac{\mathrm{d} \boldsymbol{C}^{\boldsymbol{M}}}{\mathrm{d} t}=A_{\boldsymbol{M}}(t) \boldsymbol{C}^{\boldsymbol{M}},
$$

where truncated vector of coefficients is $\boldsymbol{C}^{\boldsymbol{M}}=\left(C_{1,(1, \ldots, L)}, C_{2,(1, \ldots, L)}, \ldots, C_{K,(1, \ldots, L)}\right)^{T}$; the structure of the resulting matrix is shown in figure B.2.

We note two important properties of the system of (B.36) which are discussed in more detail in Appendix B: 
(i) For $a_{\gamma}=0$ in (1.1) the evolution of the coefficient $C_{k, l}$ in (2.18) involves only terms with $k^{\prime} \leqslant k$, i.e.,

$$
\frac{\mathrm{d}}{\mathrm{d} t} C_{k, l} \propto \sum_{k^{\prime} \leqslant k, l^{\prime}} C_{k^{\prime}, l^{\prime}}
$$

We refer to this special structure as the 'closedness' property which arises due to the properties of Hermite polynomials and orthonormality of the basis functions $\phi_{k}, \phi_{l}$ (see Appendix B and figure B.2). This degeneracy has important consequences on the evolution of the second order statistics as discussed in the next section.

(ii) The truncation directly affects the entries multiplying $C_{k-2, L+1}^{M}$ and $C_{k, L+1}^{M}$, $k=0, \ldots, K$, and the removed coefficients grow with $L$.

2.2.1. Second-order statistics from the truncated system. Given the approximate density $p^{\boldsymbol{M}}(u, \gamma, t)$ in (2.17), it is possible to compute the approximate moments of its distribution. In particular, the second-order statistics of $u$ satisfying (1.1), which is of main interest here,

$$
\bar{u}^{\boldsymbol{M}}(t)=\iint u p^{\boldsymbol{M}}(u, \gamma, t) \mathrm{d} u \mathrm{~d} \gamma, \quad \operatorname{Var}\left(u^{\boldsymbol{M}}(t)\right)=\iint(u-\bar{u})^{2} p^{\boldsymbol{M}}(u, \gamma, t) \mathrm{d} u \mathrm{~d} \gamma,
$$

can be evaluated as

$$
\bar{u}^{\boldsymbol{M}}(t)=a_{1} C_{1,0}(t), \quad \operatorname{Var}\left(u^{\boldsymbol{M}}(t)\right)=a_{2} C_{0,0}(t)+a_{3} C_{2,0}(t)-a_{1}^{2} C_{1,0}^{2}(t),
$$

where the details of the above derivations and the explicit expressions for the coefficients $a_{1}, a_{2}, a_{3}$ in terms of the system parameters are relegated to the Appendix B.

We make the following important observations which will have important consequences on the subsequent analysis (see Appendix B for a detailed discussion):

(i) The second-order statistics are determined by the three coefficients $C_{0,0}, C_{1,0}, C_{2,0}$.

(ii) For $a_{\gamma}=0$ in (1.1) and due to the closedness property (2.19) the evolution of the second-order statistics depends only on terms $C_{0, l}, C_{1, l}, C_{2, l}$ with $l=0, \ldots, L$ (see figure B.2).

We will show in Section 5 that the above properties will lead to a surprising and misleading good performance of the GrCh expansion of solutions to (1.1) when $a_{\gamma}=0$ and a spectacular failure of this method for any $a_{\gamma} \neq 0$, even when $a_{\gamma}$ is very small.

3. Exactly solvable test model with white-noise driven intermittency

The two-dimensional non-Gaussian system with quadratic nonlinearity (1.1) is arguably the simplest nontrivial exactly solvable model with intermittency and positive Lyapunov exponent where the turbulent flux due to unresolved small scale processes has important effects on the mean dynamics. Therefore, the system (1.1) serves as an ideal testbed for analyzing UQ techniques based on finitely truncated Polynomial Chaos and Gram-Charlier expansions. Bearing in mind the analogy to a turbulent high-dimensional systems, it is instructive to consider $u(t)$ in (1.1) as the 'resolved' variable which is nontrivially coupled to the unresolved process $\gamma(t)$. It is known from the previous analysis $[2,3]$ that even the simplified system (1.1) with $a_{\gamma}=0$ 
exhibits a wide range of turbulent dynamical regimes with very intermittent transient instabilities leading to fat-tailed equilibrium PDFs with only a small number of finite moments (see figure 1.1 for an illustration). Such dynamics are characteristic of the highly non-Gaussian inertial and dissipative ranges in realistic turbulent signals and we focus the subsequent analysis on various cases representing this important class of dynamical configurations.

3.1. Analytical structure of instructive configurations of the test model. We describe here four particular classes of dynamical regimes of increasing complexity which are generated by the system (1.1). These configurations are used in the following sections to analyze the performance of the PCE and GrChE as tools for uncertainty quantification in systems with white-noise driven intermittency.

Case 1: Parametric Uncertainty with time-independent Gaussian damping fluctuations

In this simple case, with parametric uncertainty leading to fat-tailed probability densities, the damping fluctuations $\gamma$ are given by a Gaussian random variable, i.e.,

$$
\gamma=\sigma_{\gamma} \xi, \quad \xi \sim \mathcal{N}(0,1),
$$

and the mean damping $\hat{\gamma}(t)$ is a known function of time, so that

$$
\dot{u}(t)=-\left(\hat{\gamma}(t)+\sigma_{\gamma} \xi\right) u(t)+f(t) .
$$

This is a configuration with non-mixing dynamics where the autocorrelation of the fluctuations is constant and equal to $\sigma_{\gamma}^{2}$. Systems in this class always have unbounded mean and variance (see Appendix E).

Case 2: Fully decoupled, exactly solvable Gaussian dynamics $\left(\boldsymbol{a}_{\boldsymbol{u}}=\boldsymbol{a}_{\boldsymbol{\gamma}}=\mathbf{0}\right)$. In this case the true dynamics of (1.1) reduce to

$$
\begin{aligned}
& \text { a) } \mathrm{d} u(t)=[-\hat{\gamma} u(t)+f(t)] \mathrm{d} t+\sigma_{u} \mathrm{~d} W_{u}(t), \\
& \text { b) } \mathrm{d} \gamma(t)=-d_{\gamma} \gamma(t) \mathrm{d} t+\sigma_{\gamma} \mathrm{d} W_{\gamma}(t)
\end{aligned}
$$

and the solutions $u(t), \gamma(t)$ are given by two independent Ornstein-Uhlenbeck processes.

Case 3: Exactly solvable non-Gaussian dynamics $\left(\boldsymbol{a}_{\boldsymbol{u}}=\mathbf{1 ,} \boldsymbol{a}_{\boldsymbol{\gamma}}=\mathbf{0}\right)$ with intermittency

In this case the dynamics of (1.1) reduce to

$$
\begin{aligned}
& \text { a) } \mathrm{d} u(t)=[-(\hat{\gamma}+\gamma(t)) u(t)+f(t)] \mathrm{d} t+\sigma_{u} \mathrm{~d} W_{u}(t), \\
& \text { b) } \mathrm{d} \gamma(t)=-d_{\gamma} \gamma(t) \mathrm{d} t+\sigma_{\gamma} \mathrm{d} W_{\gamma}(t),
\end{aligned}
$$

are conditionally linear and exactly solvable with exact formulas for the second-order statistics given in [9], and the invariant measure with fat algebraic tails can be derived analytically in the white noise limit of $\gamma(t)$. The dynamics of $\gamma(t)$ are Gaussian and decoupled from those of $u$, yet the resulting dynamics of $u(t)$ can be highly non-Gaussian and intermittent due to the quadratic nonlinearity in (3.4a). We will show in Section 4 that even an inaccurate representation of the OU process $\gamma(t)$ in (3.4) will have severe 
consequences on the approximation of the intermittent dynamics of $u(t)$. Following [2], regimes of mean-stable dynamics of the system (3.4) are (see figure 1.1 for an illustration):

(I) $\sigma_{\gamma}, d_{\gamma} \gg 1, \sigma_{\gamma} / d_{\gamma} \sim \mathcal{O}(1)$, and $\hat{\gamma}>0$ sufficiently large so that

$$
\chi_{1} \equiv-\hat{\gamma}+\frac{1}{2} \sigma_{\gamma}^{2} / d_{\gamma}^{2}<0 .
$$

This is a regime of rapidly decorrelating $\gamma(t)$. The dynamics of $u(t)$ are dominated by frequent, short-lasting transient instabilities. The decorrelation time of $u(t)$ is approximately $1 / \hat{\gamma}$ and can vary widely. This type of dynamics is characteristic of the turbulent energy transfer range.

(II) $\sigma_{\gamma}, d_{\gamma} \sim \mathcal{O}(1)$ small, $\sigma_{\gamma} / d_{\gamma} \sim \mathcal{O}(1)$, and $\hat{\gamma}>0$ sufficiently large so that $\chi_{1}<0$.

In this regime the decorrelation time of $\gamma(t)$ is long. The dynamics of $u(t)$ are characterized by intermittent bursts of large-amplitude, transient instabilities followed by quiescent phases. This regime is characteristic of the turbulent modes in the dissipative range. Similarly to (I), the decorrelation time of $u(t)$ can vary widely in this regime.

(III) $\sigma_{\gamma}^{2} / 2 d_{\gamma}^{2} \gg 1$, and $\hat{\gamma} \gg 1$ sufficiently large so that $\chi_{1}<0$. This regime is characteristic of the laminar modes in the turbulent spectrum.

Case 4: Fully coupled non-Gaussian intermittent dynamics $\left(a_{u}=1, a_{\gamma} \neq 0\right)$ In this case the general test model reduces to

$$
\begin{aligned}
& \text { a) } \mathrm{d} u(t)=(-(\hat{\gamma}+\gamma(t)) u(t)+f(t)) \mathrm{d} t+\sigma_{u} \mathrm{~d} W_{u}(t), \\
& \text { b) } \mathrm{d} \gamma(t)=-\left(d_{\gamma}+a_{\gamma} u(t)\right) \gamma(t) \mathrm{d} t+\sigma_{\gamma} \mathrm{d} W_{\gamma}(t),
\end{aligned}
$$

The equilibrium PDFs of (3.5) can be approximated numerically and they are shown in [22] to be very close to the PDFs of the system in the white noise limit. This class of dynamics will be used to illustrate the blow-up of the second order dynamics estimated from the Gram-Charlier expansions of the probability density in Section 5.

The above three configurations are used in the next two sections to study the suitability of the PCE approximations (Section 2.1) and the GrChE approximations (Section 2.2) as techniques for uncertainty quantification in systems with white noise driven intermittency. The two approaches based on the truncated spectral expansions of either the system variables or the associated joint probability density are compared with a simple Gaussian moment closure approximation (see Appendix C). Clearly, the Gaussian closure approximation is barely adequate in highly intermittent regimes but its simplicity and potential for optimization [3] make it an obvious choice for a null hypothesis. In the tests discussed in sections 4,5 we will use the unoptimized Gaussian closure approximation for the second-order statistics of the system (1.1) as the bottom line benchmark for the performance of the PCE and GrChE methods.

\section{Limitations of truncated Polynomial Chaos Expansions for systems with intermittency}

Following the general derivations of Section 2.1, here we discuss a number of cases of increasing complexity which are aimed at elucidating the properties and limitations of the PCE method for UQ in systems with white noise driven intermittency. The equations for the PCE coefficients for the test system were derived in Section 
2.1.1 and the considered examples were outlined earlier in Section 3.1. The bottom line benchmark in the tests is given by the simple Gaussian closure approximation (Appendix C). Based on the examples presented below we point out the following important limitations of the PCE method in the present context:

- For the test system (1.1) with various intermittent regimes truncated PCE performs similarly to the Gaussian closure approximation, at best (figures 4.3 and 4.4). It struggles with properly representing the second-order dynamics even in the absence of limit cycles in the dynamics (which is a common limitation in ODEs with uncertain parameters and a subject of ongoing research $[29,21,1,36,37])$.

- The pathologies in truncated PCE for intermittent dynamics stem from both the truncated Fourier-Hermite representation of white noise (see Section 2.1 and Appendix A) and from neglecting higher order coefficients in the nonlinear terms in (1.1), which become important with the onset of intermittency.

- Truncated PCE struggles with correctly representing a simple OU process (figure 4.2).

- Implementation of sparse truncation methods is hampered by a slow decay of coefficients in the PC expansions (figure 4.5).

As already mentioned earlier, these limitations are much more pronounced in systems with white-noise driven intermittency than in the context of UQ in deterministic systems with a small number of uncertain parameters when the performance of PCE tends to be much more satisfactory (e.g., [31, 11, 40, 41, 42, 43, 44, 45, 36]).

\section{PCE APPROXIMATION OF DYNAMICS WITH UNCERTAIN DAMPING}

\section{(CASE 1 OF SeCtion 3.1)}

This simple configuration is frequently used for testing PCE techniques for systems with simple parametric uncertainty in the damping fluctuations $\gamma$ of (1.1a) (e.g., [41]) which leads to fat-tailed PDFs (see figure 4.1). Contrary to the cases discussed below, the statistically exactly solvable dynamics in this configuration is non-mixing and the autocorrelation of the damping fluctuations is constant and equal to $\sigma_{\gamma}^{2}$.

The equations for the PCE coefficients reduce in this case to

$$
\dot{u}_{\alpha}=-\hat{\gamma}(t) u_{\alpha}-\sum_{p \in \mathcal{J}_{K, N}} \sum_{\substack{0 \leqslant \beta \leqslant \alpha \\ \beta+p=\alpha^{1}}} C(\alpha, \beta, p) u_{\alpha-\beta+p} \gamma_{\beta+p}+f(t) \delta_{\alpha \mathbf{0}},
$$

where $\alpha^{1}=(1,0,0, \ldots)$ and the PCE coefficients for the time-independent damping fluctuations are given by $\gamma_{\alpha^{1}}=\sigma_{\gamma}$ and $\gamma_{\alpha}=0$ for $\alpha \neq \alpha^{1}$. Here, we additionally require that

$$
\left\langle u\left(t_{0}\right)\right\rangle=1, \quad \operatorname{Var}\left(u\left(t_{0}\right)\right)=0, \quad\left\langle u\left(t_{0}\right) \gamma\right\rangle=0,
$$

which leads to the following initial conditions in (4.1):

$$
u_{\alpha^{0}}\left(t_{0}\right)=1, \quad \text { and } \quad u_{\alpha}\left(t_{0}\right)=0 \text { for } \alpha \neq \alpha^{0},
$$

where $\alpha^{0}=(0,0, \ldots)$.

We stress the following shortcomings of PCE in this simple configuration with parametric uncertainty: 

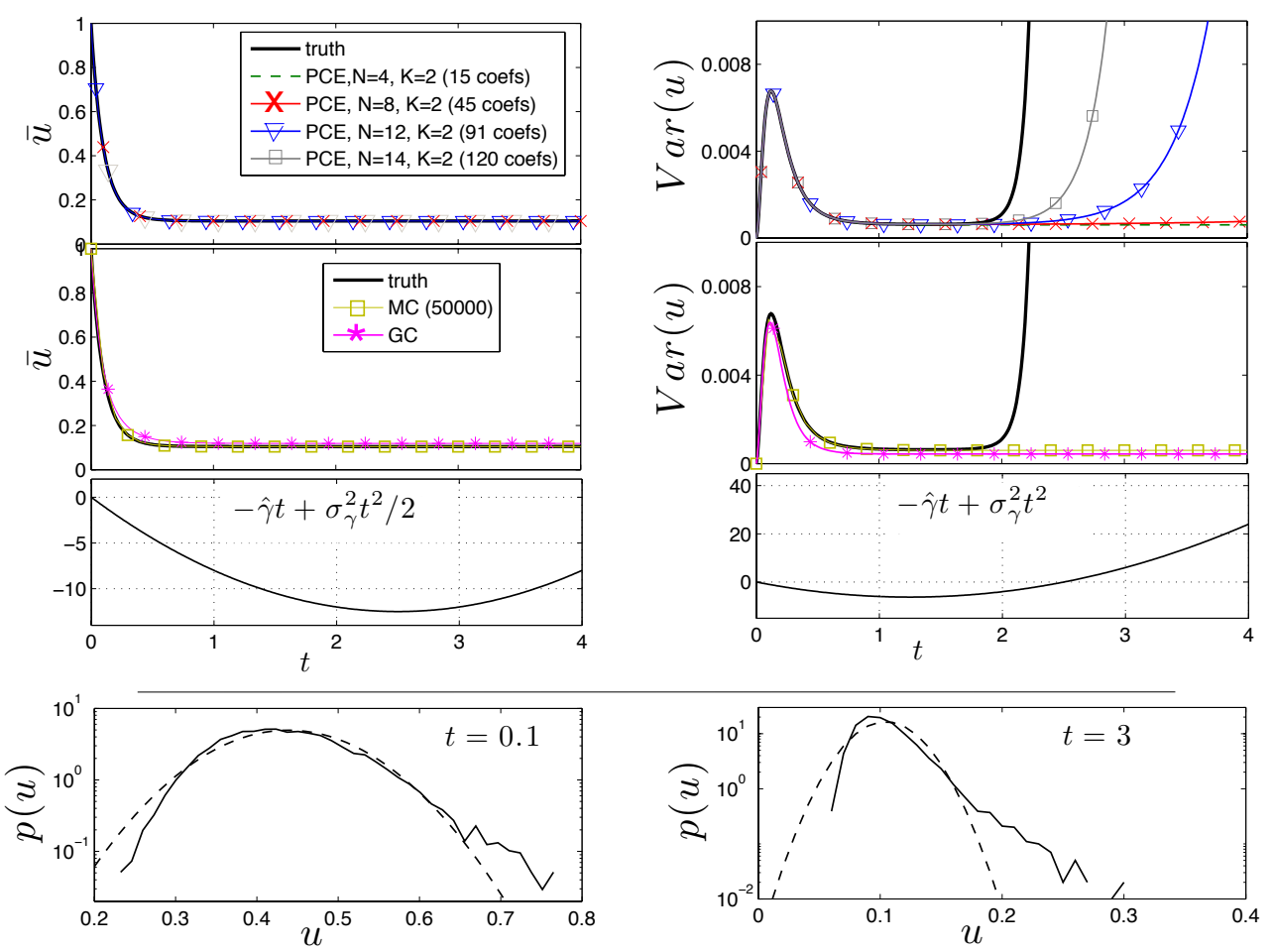

FIG. 4.1. PCE for parametric uncertainty in damping fluctuations. Truncated PCE approximation of the second-order statistics for the dynamics of $u$ in (3.2) with parametric uncertainty. Here, the damping fluctuations are represented by a Gaussian random variable $\xi \sim \mathcal{N}(0,1)$. The bottom-line benchmark is given by the Gaussian moment closure (GC). Note that both GC and the Monte Carlo simulations (with 50000 runs) fail to detect the onset of unbounded growth of the variance (see also Appendix E).

System parameters: $\hat{\boldsymbol{\gamma}}=\mathbf{1 0}, \boldsymbol{\sigma}_{\boldsymbol{\gamma}}=\mathbf{2}$, Initial conditions: $\langle u(0)\rangle=1, \operatorname{Var}(u(0))=0$, Forcing: $f(t)=1$.

- Despite a good performance of truncated PCE approximations for sufficiently short times, even very high-order truncations fail to reproduce the inevitable unbounded growth of the statistics at later times (see Appendix E); see figure 4.1 .

- The unbounded growth of the statistics of the statistics remains essentially undetected below a certain truncation order which is parameter dependent but often high (in figure 4.1 the threshold is approx. $N=9$ ); this shortcoming is also associated with the Gaussian Closure or Monte Carlo simulations.

\section{PCE approximation of a (Gaussian) ORnstein-Uhlenbeck Process} (CASE 2 OF Section 3.1)

This is the Case 2 described earlier in Section 3.1 which, despite the Gaussianity of the corresponding dynamics, is important for understanding the issues arising in PCE approximations of the non-Gaussian cases with intermittency discussed later. 
The equations for the PCE coefficients (2.10) with $\alpha \in \mathcal{J}_{K, N}$ reduce in this case to

$$
\begin{aligned}
& \text { a) } \dot{u}_{\alpha}=-\hat{\gamma} u_{\alpha}+f(t) \delta_{\alpha \mathbf{0}}+\sigma_{u} \sum_{i \in \mathcal{K}_{u}} I_{\left\{\alpha_{j}=\delta_{i j}\right\}} m_{i}(t), \\
& \text { b) } \dot{\gamma}_{\alpha}=-d_{\gamma} \gamma_{\alpha}+\sigma_{\gamma} \sum_{i \in \mathcal{K}_{\gamma}} I_{\left\{\alpha_{j}=\delta_{i j}\right\}} m_{i}(t) .
\end{aligned}
$$

Here, we consider only the Gaussian dynamics of $u$ in (4.4a) with constant forcing $f(t)=1$, and we require that

$$
\left\langle u\left(t_{0}\right)\right\rangle=0, \quad \operatorname{Var}\left(u\left(t_{0}\right)\right)=\frac{1}{2} \operatorname{Var}_{e q}(u), \quad\left\langle u\left(t_{0}\right) \dot{W}\left(t_{0}\right)\right\rangle=0,
$$

where $\operatorname{Var}_{e q}(u)=\sigma_{u}^{2} / 2 \hat{\gamma}$ is the variance of $u$ at equilibrium. The conditions (4.5) lead to the following initial conditions in (4.4):

$$
u_{\alpha^{0}}\left(t_{0}\right)=0, \quad u_{\alpha^{1}}\left(t_{0}\right)=\sqrt{\sigma_{u}^{2} / 4 \hat{\gamma}}, \quad u_{\alpha}=0 \quad \text { for } \quad \alpha \neq \alpha^{0}, \alpha^{1},
$$

where $\alpha^{0}=(0,0, \ldots), \alpha^{1}=(1,0, \ldots)$, and the truncated representation of the Wiener process is

$$
W(t)=\sum_{i=2}^{K} \xi_{i} \int_{t_{0}}^{t} m_{i}(\tau) \mathrm{d} \tau .
$$

In figure 4.2 we illustrate various truncated PCE approximations with different dimension of the random vector $\boldsymbol{\xi}$ and over time intervals of different length; first order Wick polynomials are used in all cases since the process is Gaussian.

The following properties are worth highlighting for this simple configuration:

- For sufficiently short times even low-order PCE approximations perform well for this Gaussian process.

- However, even for this Gaussian process the dimension of the random vector $\xi$ required for the desired accuracy increases very quickly with the length $T$ of the time interval over which the process is approximated. These limitations were reported earlier in $[12,19,20]$ in the context of UQ via truncated PCE approximation of the Burgers and Navier-Stokes equations driven by the white noise.

\section{PCE APPROXIMATION OF INTERMITTENT NON-GAUSSIAN DYNAMICS (CAse 3 of Section 3.1)}

In this case (see Case 3 in Section 3.1) both $u$ and $\gamma$ are driven by the white noise but only $u$ is non-Gaussian due to the quadratic nonlinearity coupling with the damping fluctuations $\gamma$. The truncated equations (2.10) for the PCE coefficients with $\alpha \in \mathcal{J}_{K, N}$ reduce in this case to

$$
\begin{aligned}
& \text { a) } \dot{u}_{\alpha}=-\hat{\gamma} u_{\alpha}-\sum_{p \in \mathcal{J}_{K, N}} \sum_{0 \leqslant \beta \leqslant \alpha} C(\alpha, \beta, p) u_{\alpha-\beta+p} \gamma_{\beta+p}+f(t) \delta_{\alpha \mathbf{0}}+\sigma_{u} \sum_{i \in \mathcal{K}_{u}} I_{\left\{\alpha_{j}=\delta_{i j}\right\}} m_{i}(t), \\
& \text { b) } \dot{\gamma}_{\alpha}=-d_{\gamma} \gamma_{\alpha}+\sigma_{\gamma} \sum_{i \in \mathcal{K}_{\gamma}} I_{\left\{\alpha_{j}=\delta_{i j}\right\}} m_{i}(t),
\end{aligned}
$$



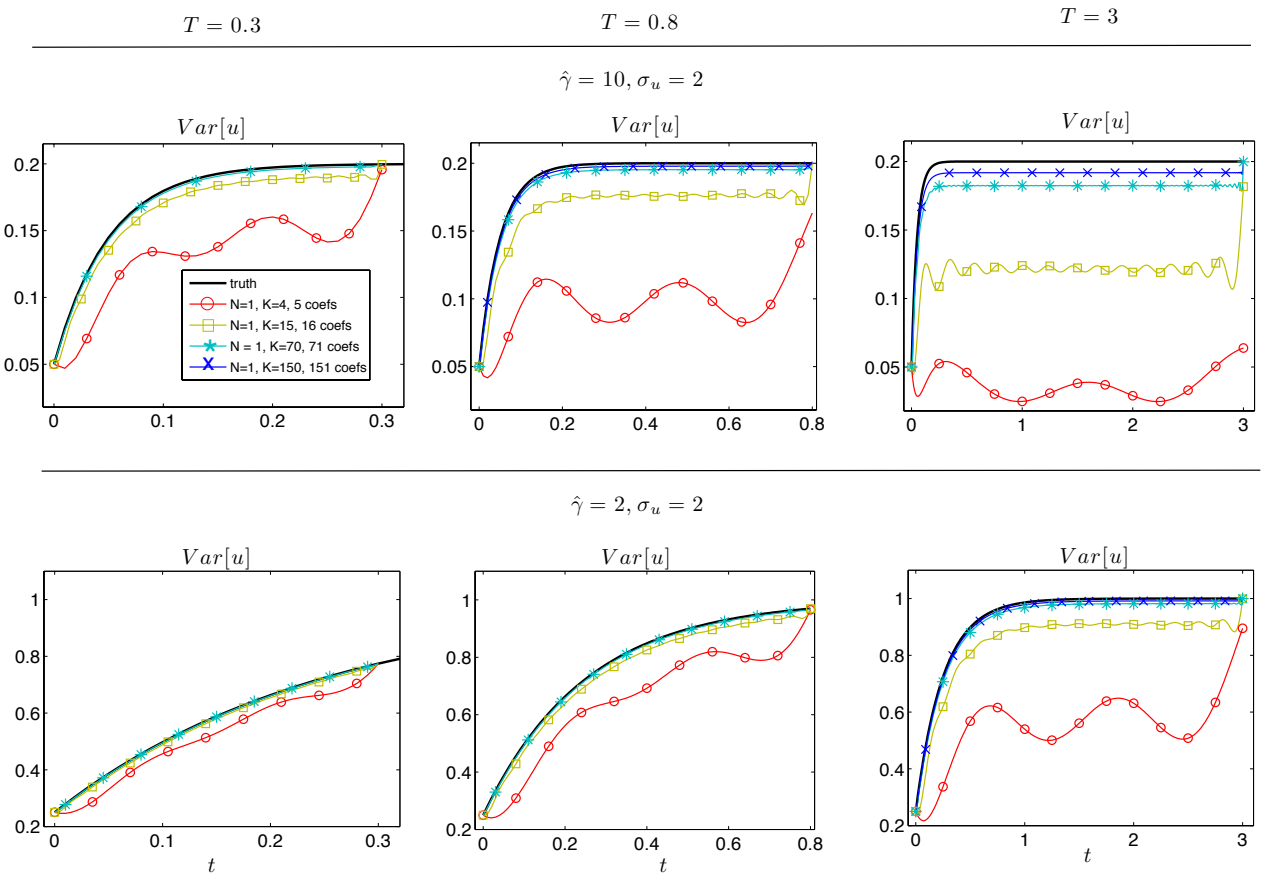

FIG. 4.2. Problematic performance of PCE approximations for a Gaussian (OrnsteinUhlenbeck) process (Case 2 of Section 3.1). Examples of PCE approximation of the second-order statistics of the Gaussian system (3.3) which is exactly solvable by an OU process. The truncated PCE approximation of various orders (see legend) is illustrated for two different values of the mean damping, $\hat{\gamma}$, and three different time intervals $[0, T]$.
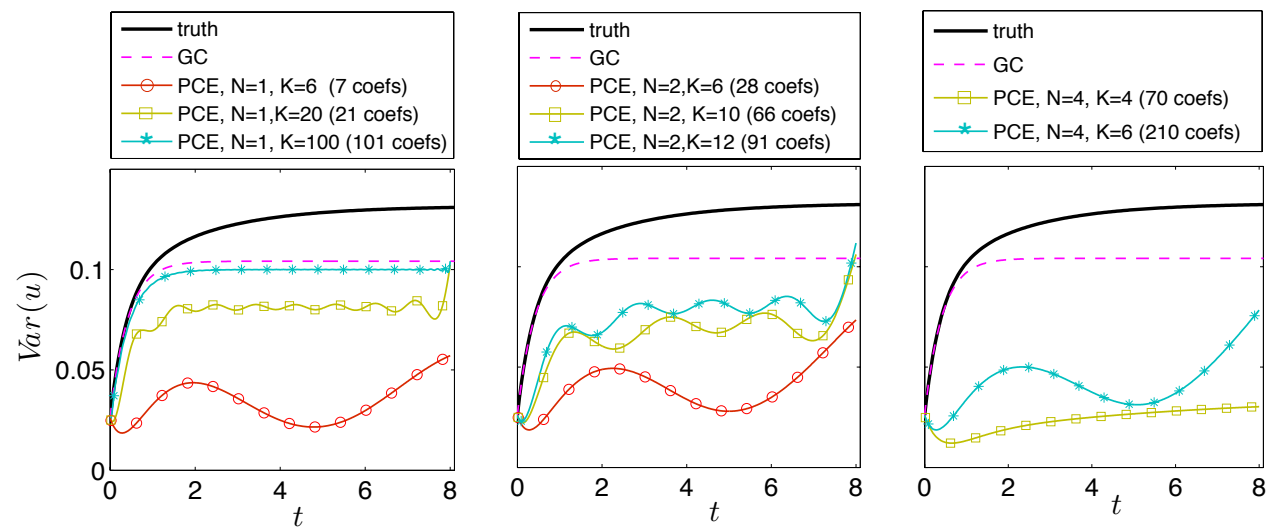

FIG. 4.3. PCE approximation of the second-order statistics in regime of intermittent large amplitude unstable bursts in (1.1), i.e., regime II of Case 3 in Section 3.1. The second-order statistics in this case can be obtained analytically (black lines) as derived in [9]. The PC approximation is illustrated for different truncations indicated in the legends; the bottom line benchmark is given by a simple Gaussian moment closure (see Appendix C).

System parameters: $\boldsymbol{a}_{\boldsymbol{u}}=\mathbf{1}, \boldsymbol{a}_{\boldsymbol{\gamma}}=\mathbf{0}, \hat{\gamma}=1.2, \sigma_{\gamma}=d_{\gamma}=0.5, \sigma_{u}=0.5$.

Forcing: $f(t)=0$

Initial condition: $p_{0}(u, \gamma)=\mathcal{N}\left(u \mid 0, \frac{1}{2} \sigma_{e q}^{u}\right) \mathcal{N}\left(\gamma \mid 0, \frac{1}{2} \sigma_{e q}^{\gamma}\right)$ where $\sigma_{e q}^{u}=\sigma_{u}^{2} / 2 \hat{\gamma}, \sigma_{e q}^{\gamma}=\sigma_{\gamma}^{2} / 2 d_{\gamma}$ are the equilibrium variances of the uncoupled $O U$ processes for $u$ and $\gamma$. 

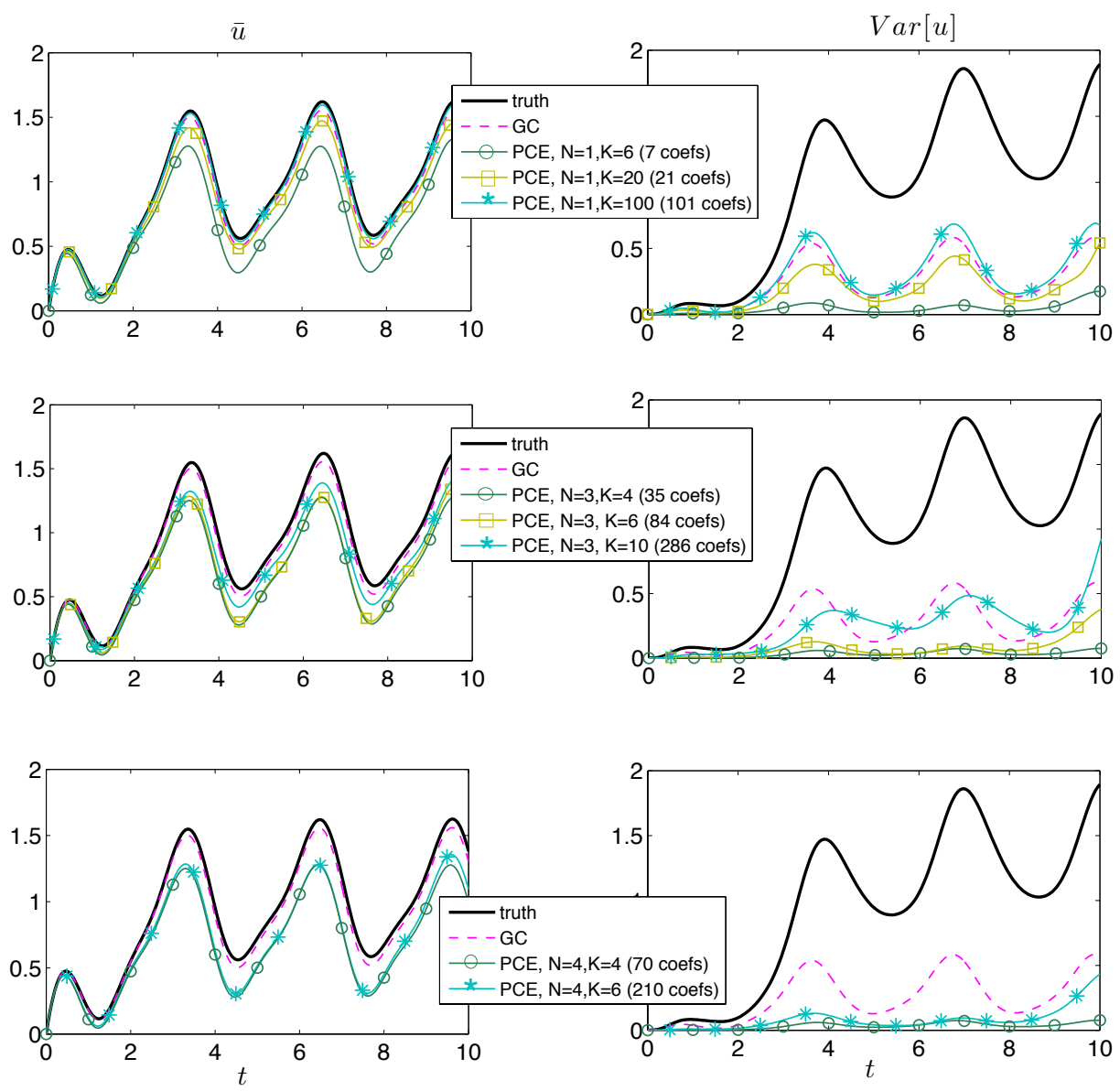

FIG. 4.4. PCE approximation of the second-order statistics in regime of frequent, short-lasting bursts of instability in (1.1); Case 3 of Section 3.1. The second-order statistics in this case can be obtained analytically (black lines) as derived in [9]. The PC approximation is illustrated for different truncations indicated in the legends; the bottom line benchmark is given by a simple Gaussian moment closure (see Appendix $C$ ).

System parameters: $\boldsymbol{a}_{\boldsymbol{u}}=\mathbf{1}, \boldsymbol{a}_{\boldsymbol{\gamma}}=\mathbf{0}, \hat{\gamma}=1.4, \sigma_{\gamma}=d_{\gamma}=10, \sigma_{u}=0.1$.

Forcing: $f(t)=A_{0}+A_{1} \cos \left(\omega_{1} t+\phi_{1}\right)+A_{2} \cos \left(\omega_{2} t+\phi_{2}\right)$ with

$A_{0}=1, A_{1}=1.1, A_{2}=0.5$
Initial condition: $p_{0}(u, \gamma)=\mathcal{N}\left(u \mid 0, \frac{1}{2} \sigma_{e q}^{u}\right) \mathcal{N}\left(\gamma \mid 0, \frac{1}{2} \sigma_{e q}^{\gamma}\right)$.

$$
A_{0}=1, A_{1}=1.1, A_{2}=0.5, \omega_{1}=2, \omega_{2}=4, \phi_{1}=1, \phi_{2}=0 .
$$

where we require that

$$
\left\langle u\left(t_{0}\right)\right\rangle=0, \quad \operatorname{Var}\left(u\left(t_{0}\right)\right)=\frac{1}{2} \operatorname{Var}_{e q}(u), \quad \operatorname{Var}\left(\gamma_{0}\right)=\frac{1}{2} \operatorname{Var}_{e q}(\gamma), \quad\left\langle u_{0} \gamma_{0}\right\rangle=0 .
$$

Moreover, we require that the white noises $\dot{W}_{u}$ and $\dot{W}_{\gamma}$, which drive $u$ and $\gamma$, are uncorrelated with each other and with the initial conditions, i.e.,

$$
\left\langle\dot{W}_{u} \dot{W}_{\gamma}\right\rangle=0, \quad\left\langle u\left(t_{0}\right) \dot{W}_{u, \gamma}\left(t_{0}\right)\right\rangle=0, \quad\left\langle\gamma\left(t_{0}\right) \dot{W}_{u, \gamma}\left(t_{0}\right)\right\rangle=0 .
$$

The constraints (4.9)-(4.10) lead to the following initial conditions in (4.8):

$$
u_{\alpha^{0}}=0, \quad u_{\alpha^{1}}=\sqrt{\sigma_{u}^{2} / 4 \hat{\gamma}}, \quad u_{\alpha^{2}}=\sqrt{\sigma_{\gamma}^{2} / 4 d_{\gamma}}, \quad u_{\alpha}=0 \quad \text { for } \quad \alpha \neq \alpha^{0}, \alpha^{1}, \alpha^{2},
$$



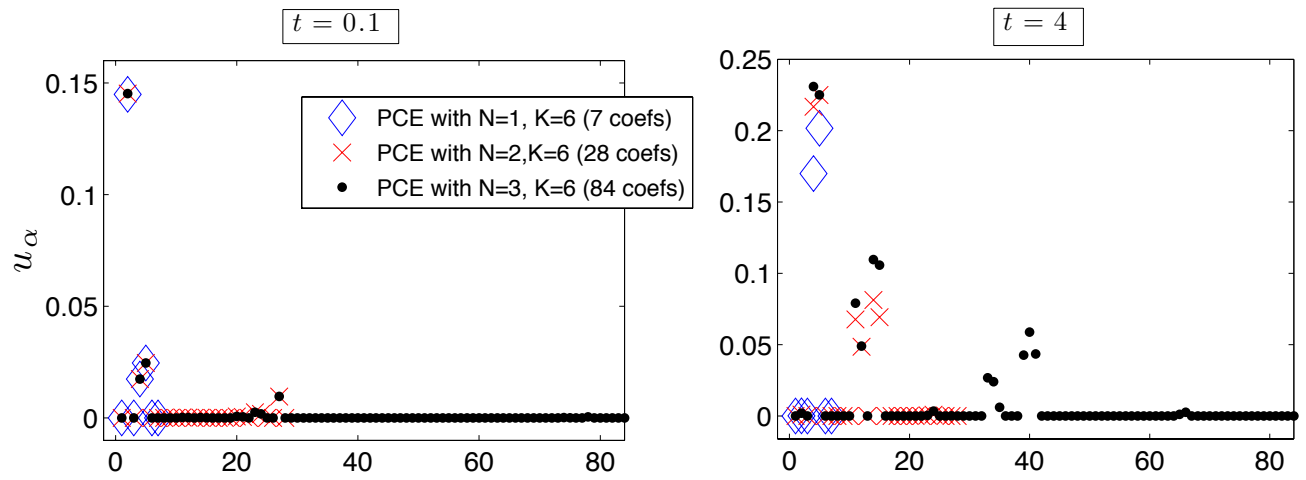

FIG. 4.5. Slow decay of PCE coefficients in the presence of white noise driven intermittency and errors due to finite truncations. The two insets show the amplitudes of the PCE coefficients at two different stages of the evolution of system (1.1) in configuration (I) of Case 3 in Section 3.1 with $\hat{\gamma}=1.2, \sigma_{u}=0.5, \sigma_{\gamma}=d_{\gamma}=10$ : nearly Gaussian dynamics (left) and highly intermittent dynamics (right) with only two finite moments. Note the non-negligible amplitudes of third order polynomials (black squares with indices $>28$ ) in the intermittent phase (right) which are absent in the lower order truncations; the sparseness is clearly still present in the distribution of the non-negligible coefficients which is not, however, as localized as in the nearly Gaussian case.

where $\alpha^{0}=(0,0, \ldots), \alpha^{1}=(1,0, \ldots), \alpha^{2}=(0,1, \ldots)$, and the truncated spectral expansions of the two independent Wiener processes (see Section 2.1 and Appendix A) are

$$
W_{u}(t)=\sum_{i \in \mathcal{K}_{u}} \xi_{i} \int_{t_{0}}^{t} m_{i}(\tau) \mathrm{d} \tau, \quad W_{\gamma}(t)=\sum_{i \in \mathcal{K}_{\gamma}} \xi_{i} \int_{t_{0}}^{t} m_{i}(\tau) \mathrm{d} \tau,
$$

where $\mathcal{K}_{u}$ and $\mathcal{K}_{\gamma}$ are two disjoint sets of integers such that $\mathcal{K}_{u} \cup \mathcal{K}_{\gamma}=\{3,4, \ldots, K\}$. Note that, compared to the previous case, roughly twice as many random variables $\xi_{i}$ are needed here to represent the Wiener processes to the same order of accuracy.

In figures 4.3 and 4.4 we show examples characteristic of dynamical configuration which show serious limitations of the truncated PCE approximations for UQ in such systems. The dynamical configuration illustrated in figure 4.3 corresponds to the regime with large amplitude intermittent instabilities in $u(t)$ (i.e., regime II in Section 3.1) and constant deterministic forcing; different time interval lengths and different order truncations are shown. Figure 4.4 shows a typical situation from the regime of abundant short lasting instabilities (i.e., regime I in Section 3.1) with time-dependent deterministic forcing.

We make the following points based on the examples illustrated in figures:

- For systems with white-noise driven intermittent regimes, truncated PCE performs, at best, similarly to the Gaussian closure approximation (figures 4.3 and 4.4). It struggles with properly representing the second-order dynamics even in the absence of limit cycles in the dynamics (which is a common limitation in ODEs with uncertain parameters.)

- In intermittent regimes both high-order of approximation (large $N$ ) and good resolution of the white noise forcing (large $K$ ) are required and PCE performs poorly, except when short time integration is carried out (see also [12]). Mod- 
erate order truncations have to compromise between the order of approximation and the resolution of the white noise forcing, resulting in either improved short or long time accuracy; in both cases PCE approximation is comparable with the simple Gaussian closure technique.

- The pathologies in truncated PCE for intermittent dynamics stem from both the finite truncation of the spectral representation of the white noise and neglecting higher order coefficients in the nonlinear terms in (1.1) which become important with the onset of intermittency.

- In the simple examples shown here, when the intermittency was due to damping fluctuations represented by an OU process, the rapidly increasing dimension of the random vector necessary for accurate approximation of this Gaussian process leads to severe difficulties in appropriately capturing the intermittency due to the white noise driven damping fluctuations even at short times.

- Contrary to nearly Gaussian dynamics, implementation of existing sparse truncation methods (e.g., $[13,35,24,8,12]$ ) is hampered in systems with white noise driven intermittency by a slow decay of expansion coefficients (figure 4.5); the sparseness is still present in the distribution of the nonnegligible coefficients but this distribution is not as localized as in the nearly Gaussian case.

\section{Limitations of truncated Gram-Charlier Expansions for systems with intermittency}

Given the truncated expansion (2.17) of the joint probability density associated with the uncertain evolution of the system (1.1), here we analyze the performance of the Gram-Charlier technique for UQ outlined in Section 2.2 and Appendix B for a number of dynamical configurations of the system (1.1) described in Section 3.1 and characterized by intermittent dynamics driven by the white noise. We examine here both the approximate densities produced by the GrChE method and the fidelity of the approximate second-order statistics, which are compared with the true statistics and estimates obtained from the simple Gaussian closure approximation outlined in Appendix C. We show the following important consequences of finite truncation of the Gram-Charlier expansions:

- The finitely truncated eigenfunction expansions lead to nonrealizability of the solutions as PDFs with the onset of nonrealizability directly linked to the onset of intermittency.

- The finite truncation leads to erroneous instabilities in the system for the coefficients in the expansion which are directly linked to nonrealizability. The erroneous instabilities lead to a blow-up of second order statistics when the true system has intermittent dynamics and $a_{\gamma} \neq 0$.

- There exists a degenerate case with $a_{\gamma}=0$ (see Case 3 of Section 3.1) when the truncated system possesses a special 'closedness property' which prevents the instabilities due to the finite truncation from down-propagating to the low-order coefficients which determine the second order statistics.

We structure this section by discussing a number of examples illustrating various limitations of the GrChE technique for UQ in systems with intermittent instabilities driven by white noise. 

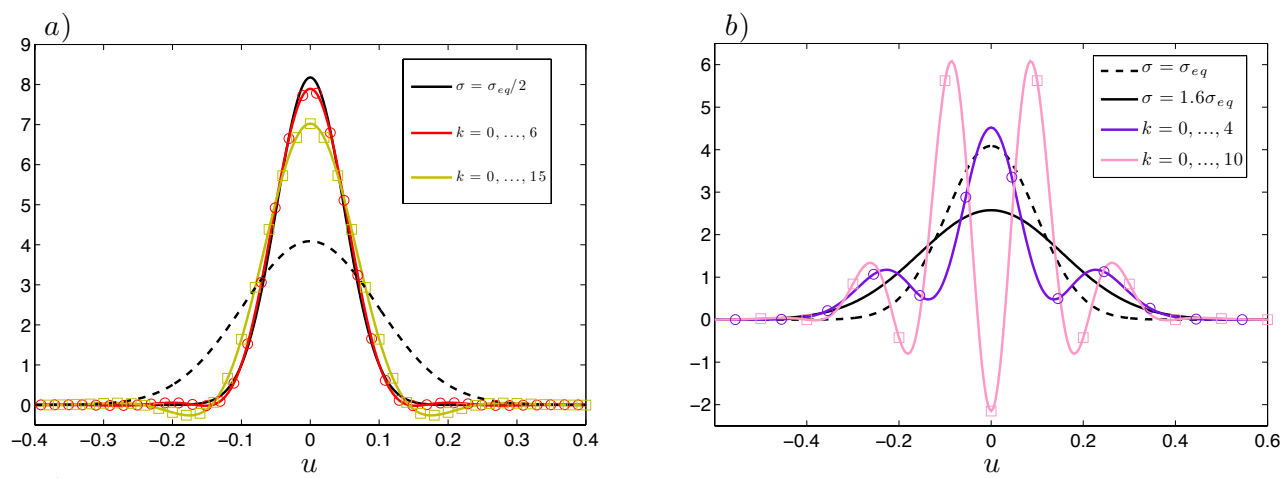

FIG. 5.1. Failure of approximations of a Gaussian density by truncated expansions in the eigenfunctions $\phi_{k}(u)$ of the Fokker-Planck operator associated with the system (1.1); see Section 2.2. (a) Approximation of a Gaussian density with a smaller variance than $\phi_{0}$ (dashed black); the true density is marked by the solid black line and the approximations are colored. (b) Approximation of a PDF with a larger variance than that of $\phi_{0}$. See Section 5 and Appendix B for more details.

\section{TRUNCATED EXPANSION OF A PROBABILITY DENSITY WITH LARGE VARIANCE}

The first symptom of limitations of the GrChE methods becomes apparent when approximating a probability density with a variance exceeding the equilibrium density of the decoupled system. In figure 5.1 we show examples of various truncated approximations of Gaussian densities with different variances. The first example illustrates the truncated approximations when the variance of the true density is smaller than that of the first eigenfunction $\phi_{0}$ (see Appendix B) in the GrCh expansion. In this case the low-order approximations are weakly nonrealizable but the accuracy of approximation quickly improves with the order of truncation. When the variance of the true distribution exceeds that of the first eigenfunction $\phi_{0}$, the finite order approximation results in spurious oscillations and nonrealizability which deteriorates with time due to erroneous exponential instabilities in the truncated system for the expansion coefficients (see Appendix B and figure B.3).

\section{NONREALIZABILITY OF TRUNCATED GRCH EXPANSIONS}

\section{AND BLOW-UP OF SECOND-ORDER STATISTICS DUE TO INTERMITTENCY}

We now discuss two examples of application of the GrChE method to estimate the second-order statistics and the marginal PDFs, $p(u, t)$, in the non-Gaussian system with intermittency (1.1) outlined earlier in Cases 3 and 4 of Section 3.1. Here, we examine both the truncated GrChE approximations for the marginal density $p(u, t)$, as well as approximations for the first two moments $\bar{u}, \operatorname{Var}[u]$ which are computed from (2.21) regardless of the possible non-realizability of the truncated approximations. Similar to the analysis of the previous section, these approximations are compared with the true marginal second-order statistics computed from formulas derived in [9] $\left(a_{\gamma}=0\right)$ or from Monte Carlo sampling $\left(a_{\gamma} \neq 0\right)$, as well as with estimates based on a simple Gaussian moment closure approximation outlined in Appendix C used as the null hypothesis. As we show below and discuss in detail in Appendix B, the exactly solvable case with $a_{\gamma}=0$ falls into a special class of degenerate systems for which the GrChE method produces surprisingly good estimates of the second-order statistics for the intermittent system (1.1) despite generating nonrealizable solutions for the approximate PDFs with spurious unbounded oscillatory terms. The sources of these 

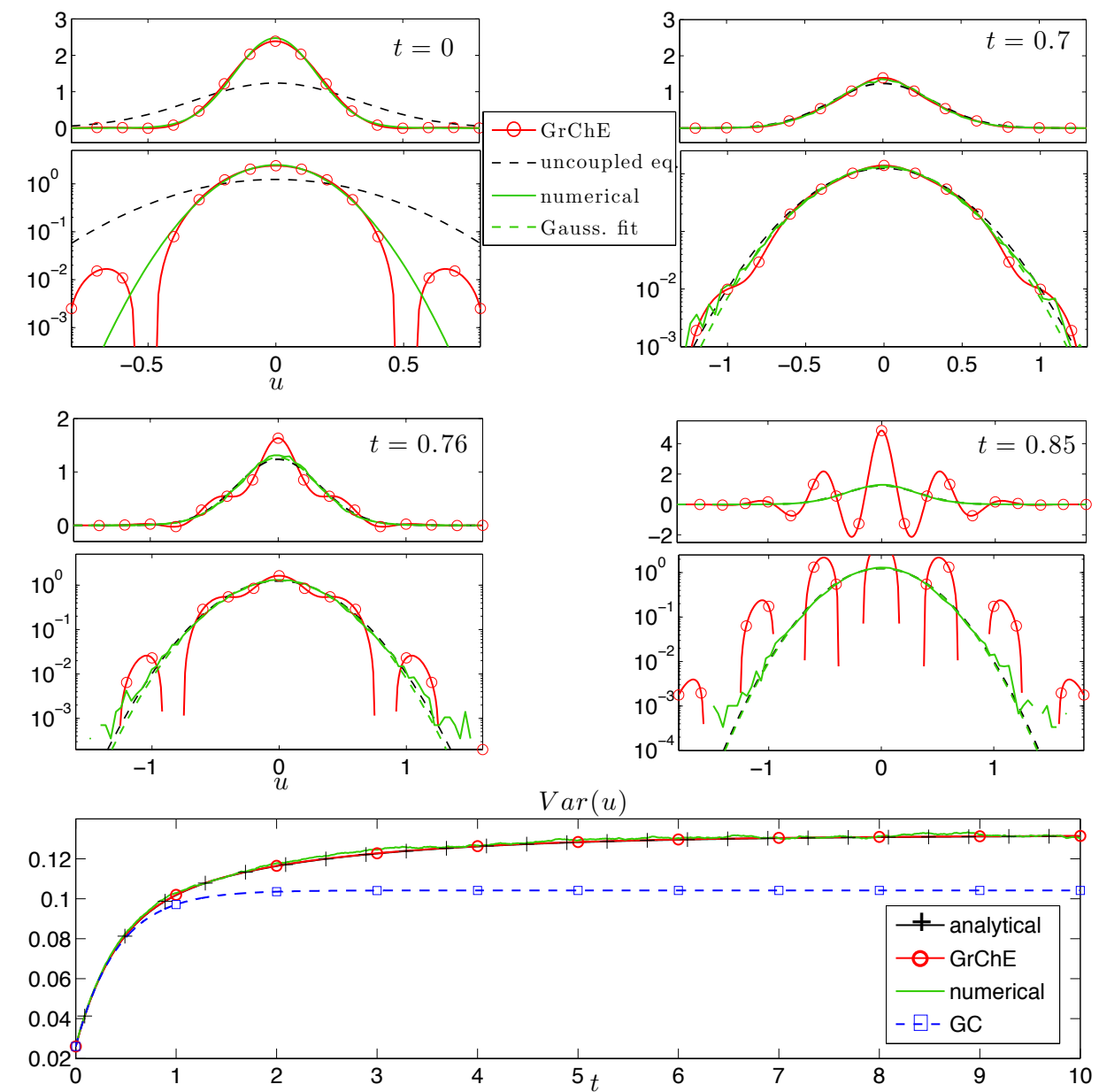

FIG. 5.2. Non-realizability of the truncated GrChE approximations (see Section 2.2 and Appendix B) for the evolution of system (1.1) with white noise driven intermittency and no deterministic forcing (regime II of Case 3 in Section 3.1). In this special case with $\boldsymbol{a}_{\boldsymbol{\gamma}}=\mathbf{0}$ in (1.1) the second-order statistics from PCE approximations are misleadingly good (see figures 5.3 and 5.4 for evolution with a very weak perturbation of this configuration).

Truncation: $K=15, L=15$.

$\lambda_{\max }=22.12$ (truncated system unstable)

System parameters: $\boldsymbol{a}=\mathbf{0}, \hat{\gamma}=1.2, \sigma_{\gamma}=d_{\gamma}=0.5, \sigma_{u}=0.5$.

Forcing: $f(t)=0$

Initial condition: $p_{0}(u, \gamma)=\mathcal{N}\left(u \mid 0, \frac{1}{2} \sigma_{e q}^{u}\right) \mathcal{N}\left(\gamma \mid 0, \frac{1}{2} \sigma_{e q}^{\gamma}\right)$ where $\sigma_{e q}^{u}=\sigma_{u}^{2} / 2 \hat{\gamma}, \sigma_{e q}^{\gamma}=\sigma_{\gamma}^{2} / 2 d_{\gamma}$ are the equilibrium variances of the uncoupled $O U$ processes for $u$ and $\gamma$.

artifacts are a direct consequence of the finite truncation and intermittency and are explained in detail in Appendix B.

Example 1. Energy dissipation regime of system (1.1) with symmetric true equilibrium PDF with fat algebraic tails (regime II of Case 3 in Section 3.1) In this regime there are very intermittent, large amplitude transient instabilities in the dynamics of $u$ which are induced by the damping fluctuations $\gamma$ with relatively 

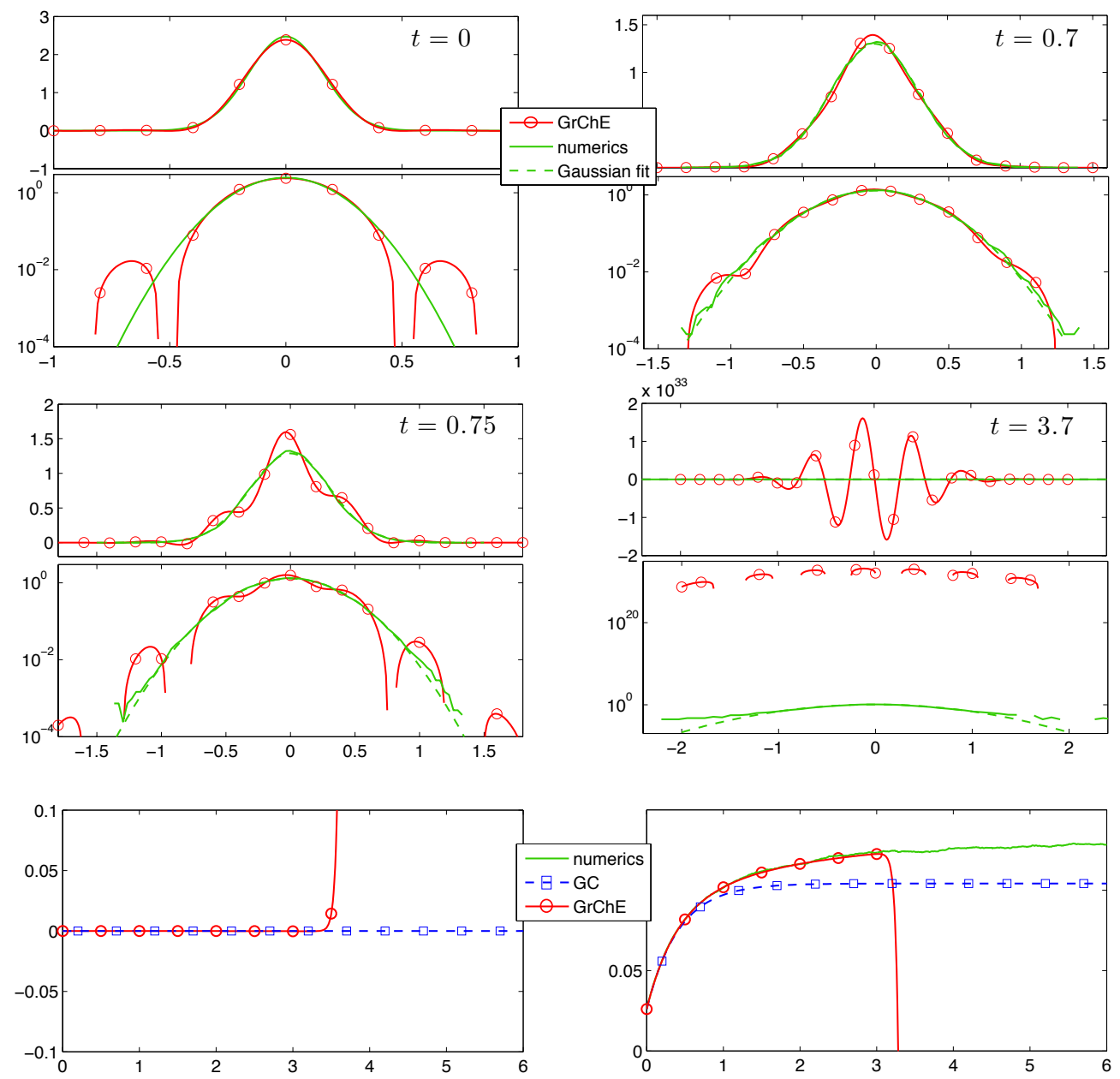

FIG. 5.3. Non-realizability of the truncated GrChE approximations (see Section 2.2 and Appendix B) and blow-up of the second-order statistics for the evolution of system (1.1) with white noise driven intermittency and no deterministic forcing (Case 4 in Section 3.1 with $\boldsymbol{a}_{\boldsymbol{u}}=\mathbf{1}, \boldsymbol{a}_{\boldsymbol{\gamma}}=\mathbf{0 . 0 1}$ corresponding to a weak perturbation of regime II from Case 3 shown in figure 5.2). The Gaussian moment closure $(G C)$ used as a bottom line benchmark is described in Appendix C.

Truncation: $K=15, L=15$.

$\lambda_{\max }=22.14$ (truncated system unstable)

System parameters: $\boldsymbol{a}=\mathbf{0 . 0 1}, \hat{\gamma}=1.2, \sigma_{\gamma}=d_{\gamma}=0.5, \sigma_{u}=0.5$.

Forcing: $f(t)=0$

Initial condition: $p_{0}(u, \gamma)=\mathcal{N}\left(u \mid 0, \frac{1}{2} \sigma_{\text {eq }}^{u}\right) \mathcal{N}\left(\gamma \mid 0, \frac{1}{2} \sigma_{\text {eq }}^{\gamma}\right)$.

broad negative minima (see also [2]). We illustrate the GrChE approximations of the system statistics in this configuration in figures 5.2-5.4 for constant non-zero forcing; these examples were chosen to highlight various artifacts arising due to the finite truncations of the $\mathrm{GrCh}$ expansions and are illustrated in the following figures:

Figure 5.2: For this special degenerate case with $a_{\gamma}=0$ in (1.1) corresponding to the exactly solvable Case 3 of Section 3.1 the truncated expansions (2.17) are nonrealizable as PDFs with spurious oscillations growing unboundedly 

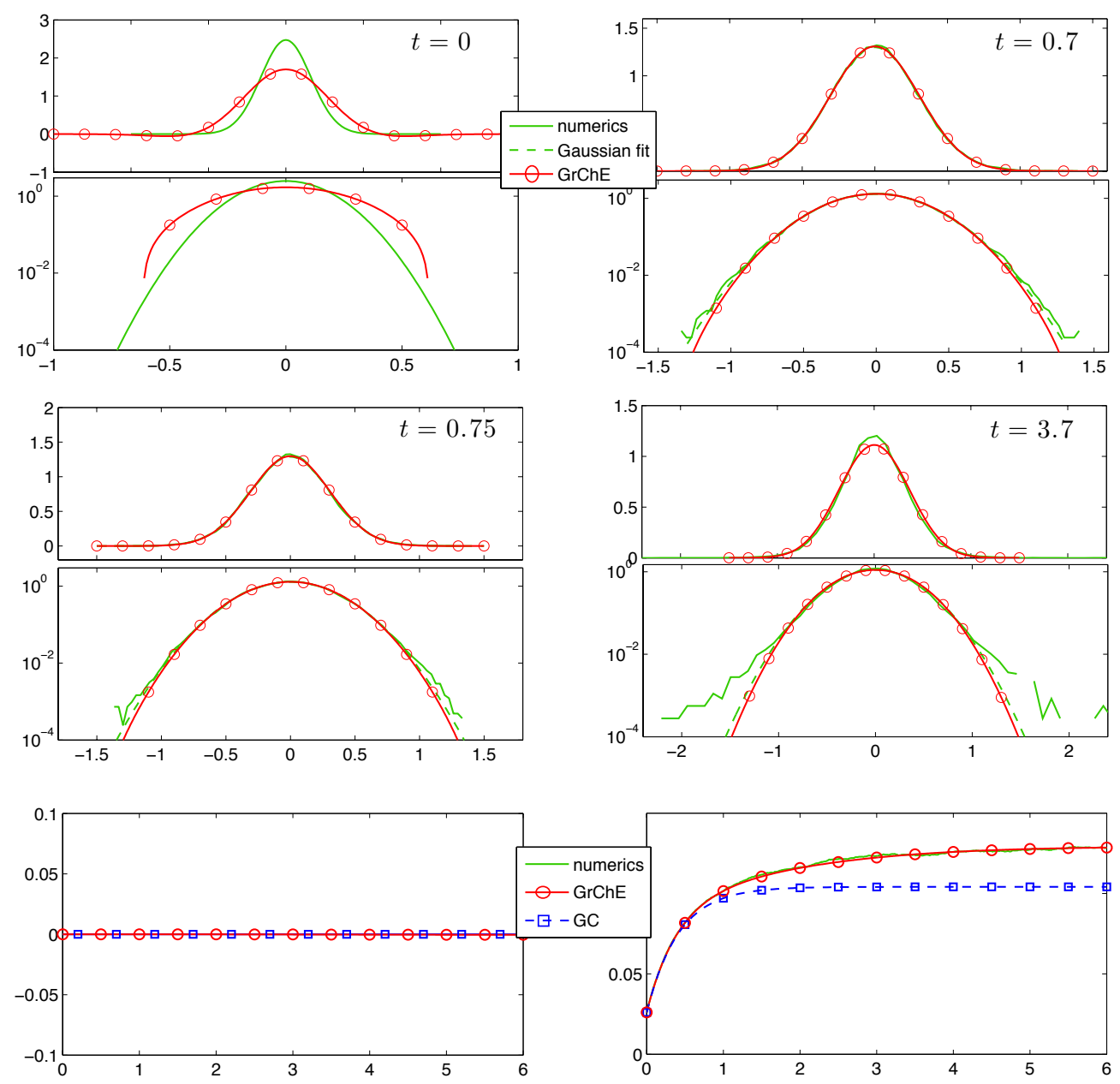

FIG. 5.4. Undetected intermittency in the true dynamics in low-order truncations of the GrChE approximations (see Section 2.2 and Appendix B) for the evolution of system (1.1) with white noise driven intermittency and no deterministic forcing (Case 4 in Section 3.1 with $\boldsymbol{a}_{\boldsymbol{u}}=\mathbf{1}, \boldsymbol{a}_{\boldsymbol{\gamma}}=\mathbf{0 . 0 1}$ corresponding to a weak perturbation of regime II from Case 3 shown in figure 5.2). The Gaussian moment closure $(G C)$ used as a bottom line benchmark is described in Appendix $C$.

Truncation: $K=3, L=3$.

$\lambda_{\max }=0$ (truncated system stable)

System parameters: $a=0.01, \hat{\gamma}=1.2, \sigma_{\gamma}=d_{\gamma}=0.5, \sigma_{u}=0.5$.

Forcing: $f(t)=0$

Initial condition: $p_{0}(u, \gamma)=\mathcal{N}\left(u \mid 0, \frac{1}{2} \sigma_{\text {eq }}^{u}\right) \mathcal{N}\left(\gamma \mid 0, \frac{1}{2} \sigma_{\text {eq }}^{\gamma}\right)$.

in time at an exponential rate; the onset of nonrealizability coincides with the onset of intermittency in the true time-dependent marginal PDFs for $u(t)$. The second-order statistics remain surprisingly accurate well beyond the blow-up of the higher moments; this is due to a particular closedness property of the system for the expansion coefficients (B.36) when $a_{\gamma}=0$, which prevents the instabilities due to the finite truncation of the GrCh expansion from downpropagating to the low order moments (see Appendix B and figures B.2 and 

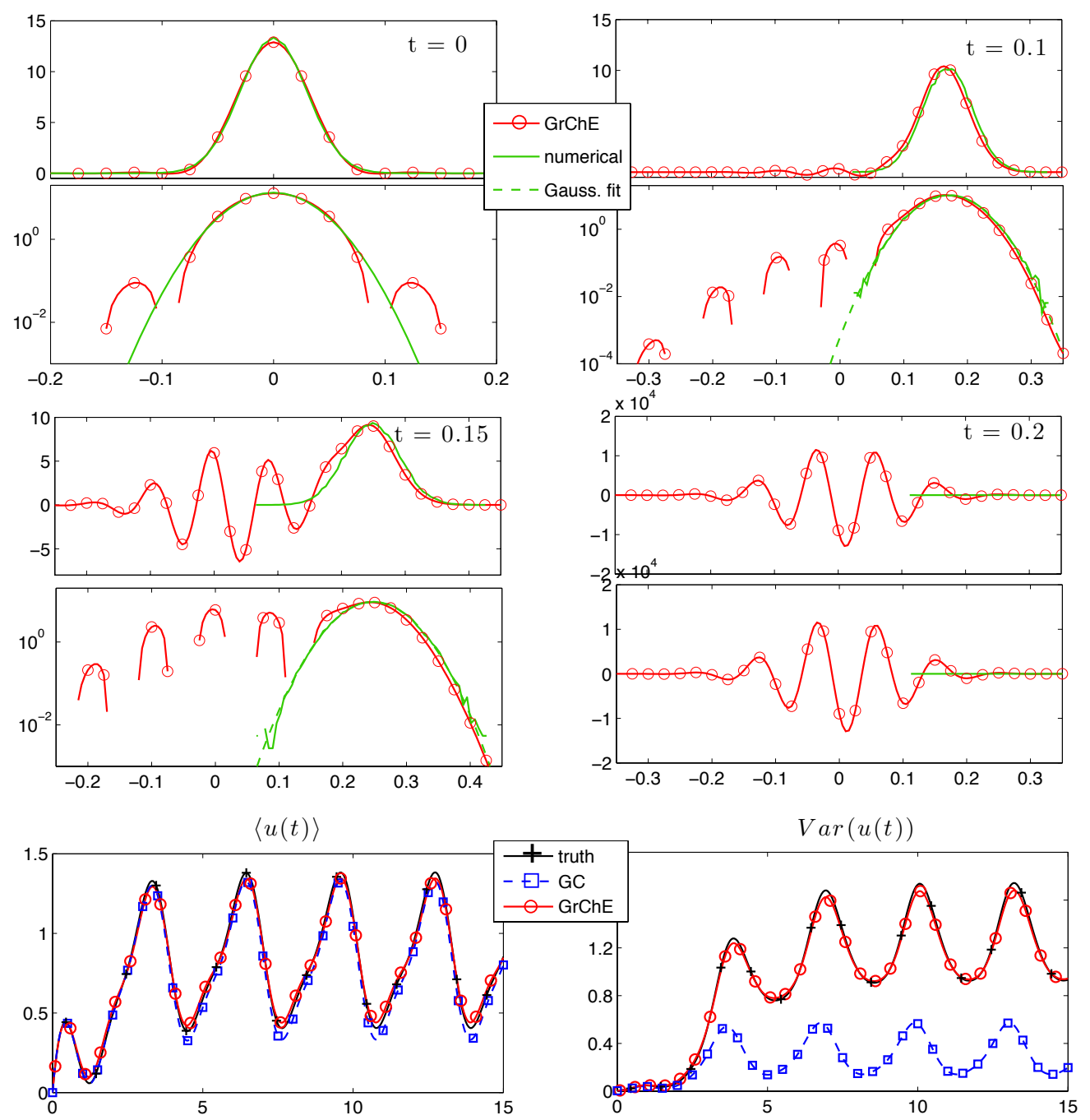

FIG. 5.5. Non-realizability of the truncated GrChE approximations (see Section 2.2 and Appendix B) for the evolution of system (1.1) with white noise driven intermittency and time-periodic forcing (regime I of Case 3 in Section 3.1). In this special case with $\boldsymbol{a}_{\boldsymbol{\gamma}}=\mathbf{0}$ in (1.1) the second-order statistics from PCE approximations are misleadingly good (see figures 5.6 and 5.7 for evolution with a very weak perturbation of this configuration).

Truncation: $K=15, L=5$.

System parameters: $\boldsymbol{a}=\mathbf{0}, \hat{\gamma}=1.4, \sigma_{\gamma}=d_{\gamma}=10, \sigma_{u}=0.1$.

Forcing: $f(t)=A_{0}+A_{1} \cos \left(\omega_{1} t+\phi_{1}\right)+A_{2} \cos \left(\omega_{2} t+\phi_{2}\right)$ with $A_{0}=1, A_{1}=1.1, A_{2}=0.5, \omega_{1}=2, \omega_{2}=4, \phi_{1}=1, \phi_{2}=0$.

Initial condition: $p_{0}(u, \gamma)=\mathcal{N}\left(u \mid 0, \frac{1}{2} \sigma_{\text {eq }}^{u}\right) \mathcal{N}\left(\gamma \mid 0, \frac{1}{2} \sigma_{\text {eq }}^{\gamma}\right)$.

B.3 for details).

Figure 5.3: Fully coupled intermittent dynamics of $u$ and $\gamma$ in (1.1), corresponding to Case 4 of Section 3.1, with a very weak coupling in the damping 

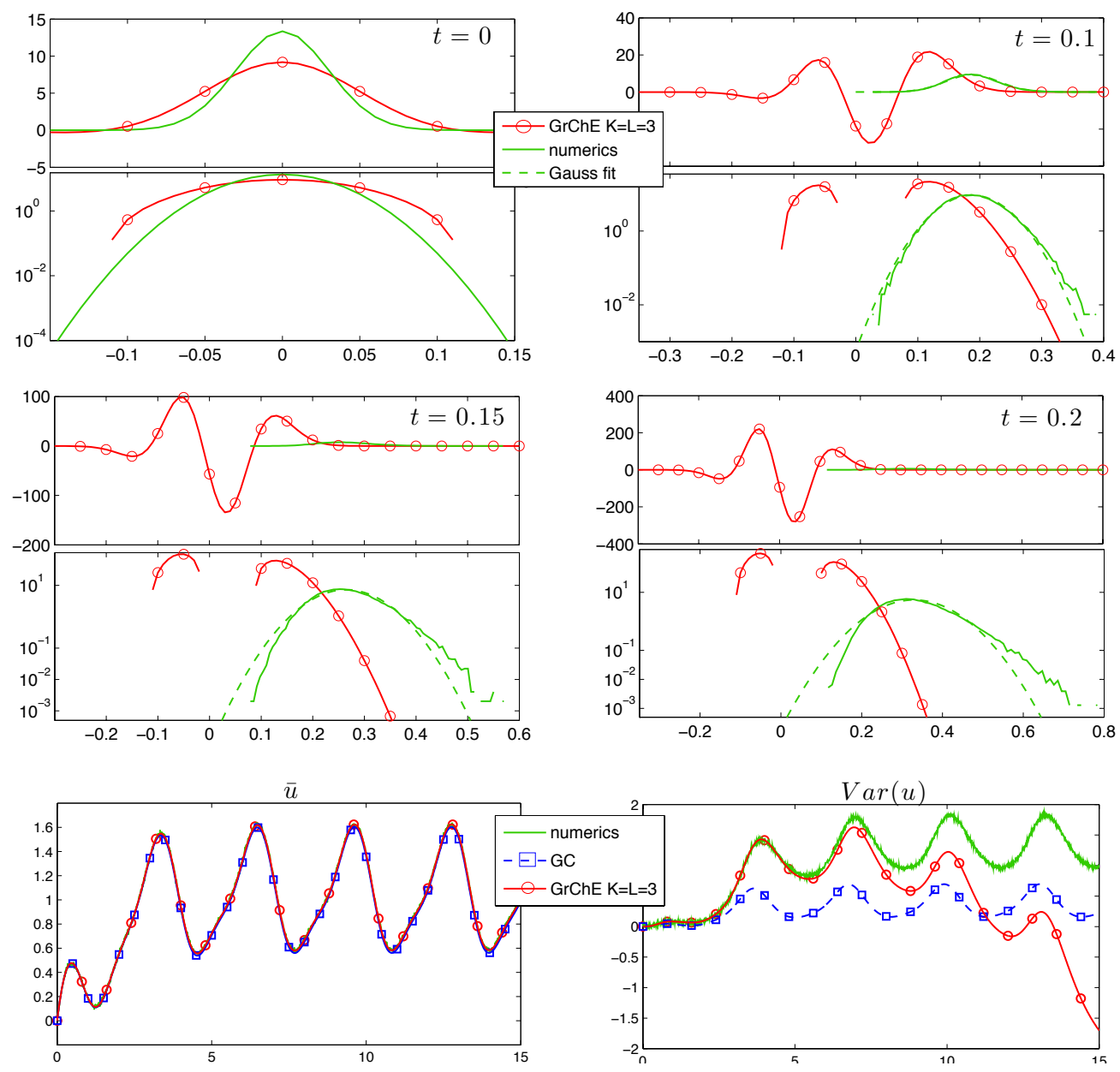

FIG. 5.6. Failure of low-order truncated GrChE approximation for the marginal density $p(u)$. Non-realizability of the truncated GrChE approximations (see Section 2.2 and Appendix $B$ ) and blow-up of variance for the evolution of system (1.1) with white noise driven intermittency and time periodic forcing (Case 4 in Section 3.1 with $\boldsymbol{a}_{\boldsymbol{u}}=\mathbf{1}, \boldsymbol{a}_{\boldsymbol{\gamma}} \mathbf{= 0 . 0 1}$ corresponding to perturbation of regime I from Case 3). The Gaussian moment closure (GC) used as a bottom line benchmark is described in Appendix $C$.

Truncation: $K=3, L=3$.

System parameters: $\boldsymbol{a}=\mathbf{0 . 0 1}, \hat{\gamma}=1.4, \sigma_{\gamma}=d_{\gamma}=10, \sigma_{u}=0.1$.

Forcing: $f(t)=A_{0}+A_{1} \cos \left(\omega_{1} t+\phi_{1}\right)+A_{2} \cos \left(\omega_{2} t+\phi_{2}\right)$ with

$A_{0}=1, A_{1}=1.1, A_{2}=0.5, \omega_{1}=2, \omega_{2}=4, \phi_{1}=1, \phi_{2}=0$.

Initial condition: $p_{0}(u, \gamma)=\mathcal{N}\left(u \mid 0, \frac{1}{2} \sigma_{\text {eq }}^{u}\right) \times \mathcal{N}\left(\gamma \mid 0, \frac{1}{2} \sigma_{\text {eq }}^{\gamma}\right)$.

fluctuations, $a_{\gamma}=0.01$. In this case the nonrealizability of the truncated GrCh expansions (2.17) is accompanied by a blow-up of the mean and variance of $u$. Here and in the subsequent cases with $a_{\gamma} \neq 0$, a word of caution is in place since, due to the lack of analytical solutions when $a_{\gamma} \neq 0$, the true statistics are approximated via Monte Carlo simulations with sample sizes between 80000 and 100000. Given the failure of MC at detecting the unbounded growth of the second order statistics in the exactly solvable case with parametric uncer- 

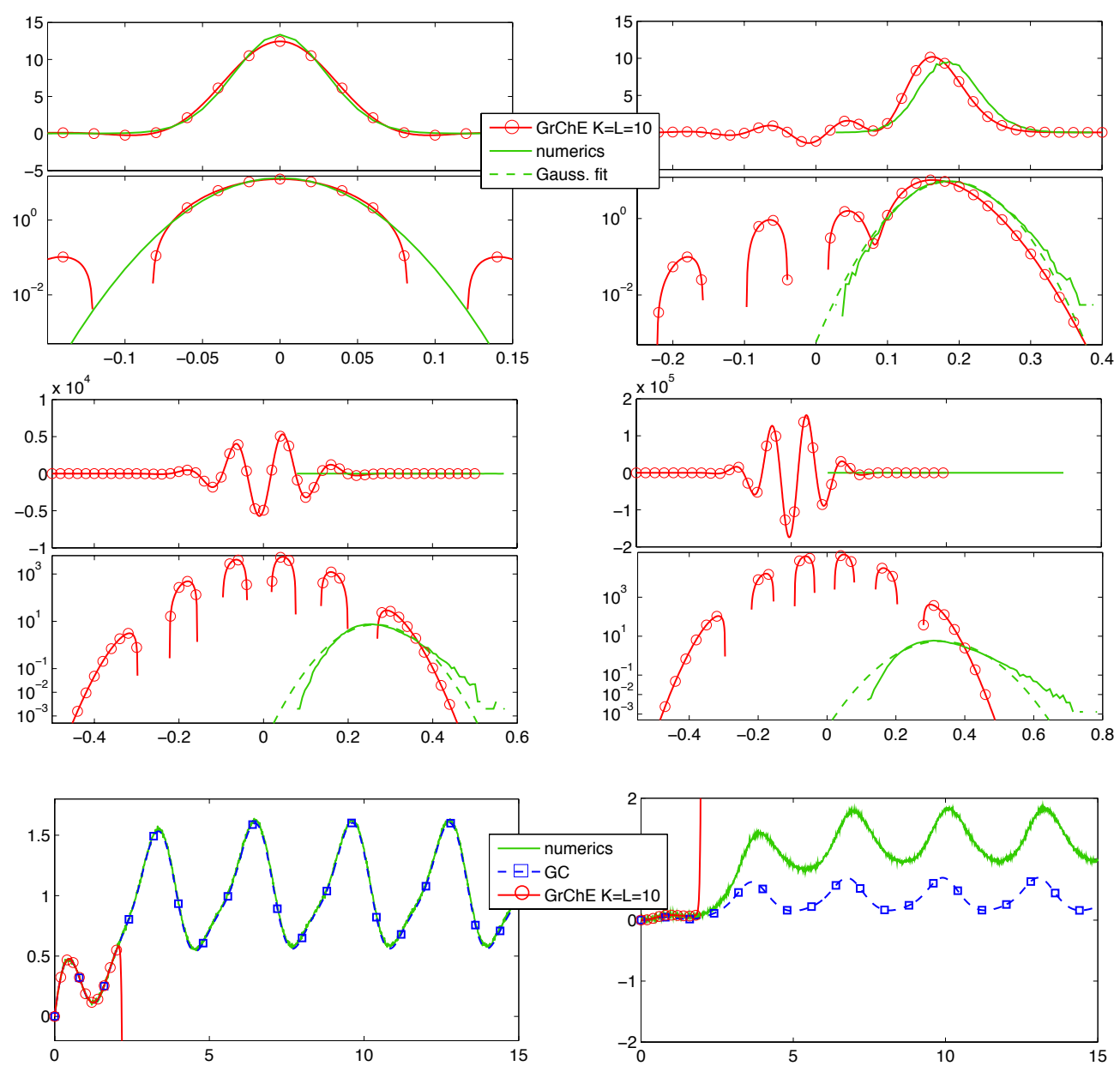

FIG. 5.7. Spectacular failure of medium-order truncated GrChE approximation for the marginal density $p(u)$. Non-realizability of the truncated GrChE approximations (see Section 2.2 and Appendix B) and blow-up of variance for the evolution of system (1.1) with white noise driven intermittency and time periodic forcing (Case 4 in Section 3.1 with $\boldsymbol{a}_{\boldsymbol{u}}=\mathbf{1} \boldsymbol{a}_{\boldsymbol{\gamma}}=\mathbf{0 . 0 1}$ corresponding to perturbation of regime I from Case 3). The Gaussian moment closure (GC) used as a bottom line benchmark is described in Appendix $C$.

Truncation: $K=10, L=10$.

System parameters: $\boldsymbol{a}=\mathbf{0 . 0 1}, \hat{\gamma}=1.4, \sigma_{\gamma}=d_{\gamma}=10, \sigma_{u}=0.1$.

Forcing: $f(t)=A_{0}+A_{1} \cos \left(\omega_{1} t+\phi_{1}\right)+A_{2} \cos \left(\omega_{2} t+\phi_{2}\right)$ with

$A_{0}=1, A_{1}=1.1, A_{2}=0.5$,
Initial condition: $p_{0}(u, \gamma)=\mathcal{N}\left(u \mid 0, \frac{1}{2} \sigma_{e q}^{u}\right) \times \mathcal{N}\left(\gamma \mid 0, \frac{1}{2} \sigma_{e q}^{\gamma}\right)$.

tainty (figure 4.1), we cannot fully exclude the possibility of the MC missing the important rare events. In any case this does not affect the issue of the nonrealizability of the $\mathrm{GrChE}$ approximations.

Figure 5.4: Fully coupled intermittent dynamics of $u$ and $\gamma$ in (1.1), as in figure 5.4, but for a very low-order GrCh expansion. In this case the truncated expansions lead to realizable PDFs and produce good second-order statistics, but the intermittency apparent in the dynamics of the true system is 
completely missed in the GrChE approximations.

Example 2. Energy transfer regime, true time-dependent PDF on attractor skewed with fat algebraic tails. In this regime (regime I of Section 3.1) there are very frequent transient instabilities in the dynamics of $u$ which are induced by the damping fluctuations $\gamma$ with abundant relatively narrow negative minima (see also [2]). The GrChE approximations for the marginal density $p(u, t)$ and the first two moments are shown in figures 5.5-5.7 for time-periodic forcing; all these examples show a very quick onset of nonrealizability and a blow-up of the second-order statistics except in the special case $a_{\gamma}=0$ when the damping fluctuations $\gamma(t)$ are fully decoupled from $u(t)$. Three figures illustrating these erroneous effects are:

Figure 5.5: Similarly to Example 1, in this special configuration of (1.1) with $a_{\gamma}=0$ (exactly solvable Case 3 of Section 3.1) the truncated expansions (2.17) are nonrealizable as PDFs with spurious oscillations growing unboundedly in time at an exponential rate; the onset of nonrealizability coincides with the onset of intermittency in the true time-dependent marginal PDFs for $u(t)$. The second-order statistics remain surprisingly accurate well beyond the blow up of the higher moments; this is due to a particular closedness property of the system for the expansion coefficients (B.36), which prevents the instabilities due to truncation from down-propagating to the low order moments (see Appendix B for details).

Figure 5.6: Low-order truncation of $\mathrm{GrCh}$ expansion (2.17) of fully coupled intermittent dynamics of $u$ and $\gamma$ in (1.1), corresponding to Case 4 of Section 3.1, with a very weak coupling in the damping fluctuations, $a_{\gamma}=0.01$. Contrary to the similar case discussed in Example 1, the low-order statistics diverge here even for low order truncations; the truncated expansions are non-realizable and completely miss the skewness of the true time-dependent PDF.

Figure 5.7: Higher-order truncation of GrCh expansion (2.17) of fully coupled intermittent dynamics of $u$ and $\gamma$ in (1.1) with a very weak coupling in the damping fluctuations, $a_{\gamma}=0.01$. Here, the mean and variance blow up at short times and are accompanied by non-realizability with intermittency of the true system completely missed.

Given the examples shown in figures 5.2-5.7, we reiterate below the main points concerning the limitations of the GrChE method for UQ in systems with white noise driven intermittency:

- FUlLy COUPLED NON-GAUSSIAN INTERMITTENT DYNAMICS $\left(a_{u}, a_{\gamma} \neq 0\right.$ in (1.1) corresponding to Case 4 of Section 3.1; figures 5.3, 5.4, 5.6, and 5.7)

- Nonrealizability of the marginal PDFs for $u$ and a blow-up of the secondorder statistics, even for $a_{\gamma} \ll 1$ in (1.1). Exception: very low order truncation, $K, L \lesssim 3$ when good second-order statistics are usually achieved but intermittency is completely missed.

- The nonrealizability of the marginal PDFs for $u$ coincides with the onset of intermittency.

- Erroneous exponential instabilities in the truncated system (B.36) for the expansion coefficients which grow with increasing truncation order and increasing intermittency (figure B.3). The finite Galerkin truncation of the system for the expansion coefficients $C_{k, l}^{M}$ of the probability density $p(u, \gamma)$ induces a loss negative semi-definiteness of the system matrix 
for sufficiently small mean damping $\hat{\gamma}$ and sufficiently large truncation order.

- Spectacular failure of truncated eigenfunction expansions for timeperiodic forcing (figures 5.6 and 5.7).

- False equilibrium solutions. The solutions of the truncated system (1.1) with intermittency lead to erroneous, often non-realizable, approximations for the probability densities even in the absence of the finitetruncation instability.

The nonrealizability of the approximations obtained from the truncated eigenfunction expansions of $p(u, \gamma, t)$ occurs for:

- breaking the symmetry by introducing non-zero forcing in (1.1),

- sufficiently small mean damping $\hat{\gamma}$ which is associated with the onset of intermittency,

- sufficiently small noise amplitude $\sigma_{u}$,

- sufficiently large noise amplitude $\sigma_{\gamma}$ and damping $d_{\gamma}$ in $\gamma(t)$,

- sufficiently high order truncation (usually $K, L>5$ is sufficient).

\section{- EXACTLY SOLVABLE NON-GAUSSIAN INTERMITTENT DYNAMICS}

$\left(a_{u} \neq 0, a_{\gamma}=0\right.$ in (1.1) corresponding to Case 3 of Section 3.1; figures 5.2 and 5.5$)$

- Nonrealizability of the truncated approximations for the densities and erroneous exponential instabilities as in the general case above.

- Unexpectedly good approximation of the marginal second-order statistics, $\bar{u}, \operatorname{Var}(u)$, for the system (1.1) from truncated eigenfunction expansions regardless of truncation order (figures 5.2 and 5.5). The instabilities due to finite truncation do not down-propagate to the lower order modes because of the 'lower triangular' structure of the truncated system matrix (see figure B.2b) resulting in closed equations for approximate marginal mean and variance.

\section{Conclusions}

In Section 3 we introduced a simple unambiguous test problem for UQ in systems with transient intermittent instability, and for UQ in systems with parametric uncertainty in the damping leading to fat-tailed probability densities. We then examined the suitability of truncated Polynomial Chaos Expansions (PCE) and truncated Gram-Charlier Expansions (GrChE) as possible methods for uncertainty quantification in nonlinear systems with positive Lyapunov exponents due to either the intermittency or damping uncertainties; such systems are commonly encountered in Atmosphere and Ocean Science applications dealing with turbulent signals from highdimensional systems. The two methods examined here rely on truncated Galerkin projections of either the system variables in a fixed random polynomial basis (PCE) or a suitable eigenfunction expansion of the joint probability distribution associated with the uncertain evolution of the system (GrChE). Based on the simple, unambiguous and statistically exactly solvable non-linear and non-Gaussian test model, we showed that methods exploiting truncated spectral expansions, be it PCE or GrChE, have significant limitations for uncertainty quantification in systems with intermittent instabilities or parametric uncertainties in the damping even in the absence of explicit time dependence or nontrivial attractors in the deterministic part of dynamics; these are known problems for UQ based on PCE in simpler systems with a finite number 
of uncertain parameters. Intermittency and fat-tailed PDFs are hallmark features of the inertial and dissipation ranges of turbulence and we show that in such important dynamical regimes PCE performs, at best, similarly to the simple Gaussian moment closure technique utilized earlier by the authors for UQ within a framework of Empirical Information Theory [3]. Moreover, we show that the non-realizability of the $\mathrm{GrChE}$ approximations for probability densities is linked to the onset of intermittency in the dynamics and it is frequently accompanied by an erroneous blow-up of the second-order statistics at short times. The limitations discussed here in an unambiguous simple test model are similar to those encountered in earlier heuristic applications to turbulence theory and stem from the following:

- Non-uniform convergence in time of PCE and GrChE resulting in a rapidly increasing number of terms necessary for a good approximation of the random process as time evolves.

- Fundamental problems with capturing the constant flux of randomness due to the white noise forcing via finite truncations of the Fourier-Hermite expansion of the associated Wiener process.

- Slow decay of PCE and GrChE coefficients in the presence of intermittency which substantially complicates development of sparse truncation methods which have been widely used in nearly elliptic problems or in low Reynolds number flows.

Rigorous derivations were richly illustrated throughout the paper by various straightforward tests exploiting the simple two-dimensional, nonlinear, and non-Gaussian model with intermittency and a positive Lyapunov exponent. The combination of analytical tractability and a very rich dynamical behavior of the test model (1.1) used in this study represents a very attractive testbed for analyzing the performance of various techniques for uncertainty quantification in turbulent dynamical systems. We therefore propose this model as a challenging, yet mathematically tractable, benchmark for testing future methods for UQ in the important class of systems with intermittency.

The results presented in this paper point to a number of fundamental limitations of both the PCE and GrChE approaches to UQ in systems with intermittency, or systems with fat-tailed PDFs due to parametric uncertainties, which are summarized below.

Limitations of PCE approximations of systems with parametric uncertainty in the damping:

- Despite the good performance of truncated PCE approximations for UQ in such systems for sufficiently short times, even very high-order truncations often fail to reproduce the unbounded growth of the statistics at later times (figure 4.1).

Limitations of PCE approximations of uncertain dynamics with intermittency:

- For the test model (1.1) with various realistic intermittent regimes, the truncated PCE performs, at best, similarly to the Gaussian closure approximation (figures 4.3 and 4.4). It struggles with properly representing the statistics of uncertain dynamics with intermittency even in the absence of limit cycles, which is a common limitation in ODEs with uncertain parameters.

- The pathologies in truncated PCE for intermittent dynamics stem from both the finite spectral truncation of the white noise expansion in Fourier-Hermite series and from neglecting higher order coefficients in the nonlinear terms in (1.1) which become important with the onset of intermittency (figure 4.5). 
- Truncated PCE struggles with correctly representing a simple Gaussian (OU) process (figure 4.2), except at short times or for small amplitudes of the white noise forcing (see also [12] for additional discussion and error bounds).

- Implementation of existing sparse truncation methods (e.g., [13, 35, 24, 8]) is hampered by a slow decay of the expansion coefficients (e.g., figure 4.5).

Limitations of the GrChE approximations of the joint probability density in systems with intermittency:

- The finitely truncated eigenfunction expansions of probability densities lead to nonrealizability of the approximations with the onset of nonrealizability directly linked to the onset of intermittency (figures 5.2-5.7).

- The finite truncation of $\mathrm{GrCh}$ expansions leads to erroneous instabilities in the system for the expansion coefficients which are directly linked to nonrealizability (see figure B.3 and Appendix B). The erroneous instabilities frequently lead to a blow-up of the second-order statistics in regimes of intermittent dynamics (see figures 5.3, 5.6, and 5.7).

The above shortcomings of the two methods clearly indicate that further work is in order for a reliable UQ in turbulent systems with white noise driven intermittency. However, given the history of the subject, new approaches are likely to improve the performance of truncated spectral expansions in such dynamical configurations. Regardless of future developments, the improved PCE techniques would necessarily have to maintain a reasonably small number of the random basis polynomials while adequately capturing the effects of the white noise forcing. In this context some hybrid methods [12] combining solutions from a sufficiently fine partition of the time interval of interest might offer a possible direction for improvements, but the issue seems far from resolved. The truncated Gram-Charlier expansions of probability densities which do not suffer from artifacts due to misrepresentation of the white noise forcing are, in general, severely affected by the onset of intermittency associated with fattailed densities with few finite moments; here, appropriate techniques for reordering the expansion terms (e.g., [14]) might prove useful. However, any developments in this area are yet to be tested.

Acknowledgments. This research of A.J.M. is partially supported by National Science Foundation grant DMS-0456713 and the office of Naval Research grants ONR DRI N0014-10-1-0554 and N00014-11-1-0306. M.B is supported as a postdoctoral fellow on the first ONR grant.

Appendix A. Polynomial Chaos Expansion of the test system with intermittency. Below we complement the presentation of Section 2.1 with a few more technical details and relevant theorems which lay foundations for the Polynomial Chaos Expansion technique and build on the original ideas of the Homogeneous Chaos of Wiener [38].

Recall that the Wick polynomials were defined in Section 2.1 as

$$
T_{\alpha}(\boldsymbol{\xi})=\prod_{i=1}^{\infty} H_{\alpha_{i}}\left(\xi_{i}\right),
$$

where $H_{n}$ is the normalized $n$-th order Hermite polynomial and $\alpha_{i}$ belong to a set of 
multiindices with a finite number of nonzero components

$$
\mathcal{J}=\left\{\alpha=\left(\alpha_{i}, i \geqslant 1 \mid \alpha_{i} \in\{0,1,2, \ldots\}\right),|\alpha|=\sum_{i=1}^{\infty} \alpha_{i}<\infty\right\},
$$

so that the product in the right hand side of (A.1) has a finite number of factors and is therefore well defined. Wick polynomials are defined over an infinite dimensional space with variables $\boldsymbol{\xi}=\left(\xi_{1}, \xi_{2}, \ldots\right)$, where $\left\langle\xi_{i}\right\rangle=0,\left\langle\xi_{i} \xi_{j}\right\rangle=\delta_{i j}$, and they form a complete orthonormal basis in $L^{2}$ on the probability space with respect to the Gaussian measure generated by $\xi$. In particular

$$
\left\langle T_{\alpha} T_{\beta}\right\rangle=\delta_{\alpha \beta}, \quad\left\langle T_{0}\right\rangle=1, \quad\left\langle T_{\alpha}\right\rangle=0 \text { when } \alpha \neq 0 .
$$

The order of the Wick polynomial $T_{\alpha}$ is defined as $|\alpha|=\sum \alpha_{i}$.

The following result, often referred to as the Cameron-Martin Theorem, forms the foundation of Wiener Chaos theory.

Theorem 1 (Cameron-Martin [4]) Assume that for fixed $x$ and $s \leqslant t, u(x, s)$ is a functional of the Wiener process $W$ on the interval $[0, s]$ with $\left\langle|u(x, s)|^{2}\right\rangle<\infty$, then $u(x, s)$ has the following Fourier-Hermite expansion:

$$
u(x, s)=\sum_{\alpha \in \mathcal{J}} u_{\alpha}(x, s) T_{\alpha}, \quad u_{\alpha}(x, s)=\left\langle u(x, s) T_{\alpha}\right\rangle,
$$

where $T_{\alpha}$ are the Wick polynomials defined by (A.1). Furthermore, the first two statistical moments of $u(x, s)$ are given by

$$
\langle u(x, s)\rangle=u_{0}(x, s)
$$

and

$$
\left\langle|u(x, s)|^{2}\right\rangle=\sum_{\alpha \in \mathcal{J}}\left|u_{\alpha}(x, s)\right|^{2} .
$$

The following theorem due to [27] is very useful for our analysis since it allows for exact treatment of nonlinearities.

Theorem 2. Suppose $u, \gamma$ have Wiener Chaos expansions

$$
u=\sum_{\alpha \in \mathcal{J}} u_{\alpha} T_{\alpha}(\xi), \quad \gamma=\sum_{\beta \in \mathcal{J}} \gamma_{\alpha} T_{\beta}(\xi) .
$$

If $\left\langle|u v|^{2}\right\rangle<\infty$, then the product $u v$ has the Wiener Chaos expansion

$$
u v=\sum_{\theta \in \mathcal{J}}\left(\sum_{p \in \mathcal{J}} \sum_{0 \leqslant \beta \leqslant \theta} C(\theta, \beta, p) u_{\theta-\beta+p} \gamma_{\beta+p}\right) T_{\theta}(\xi)
$$

where

$$
C(\theta, \beta, p)=\left[\left(\begin{array}{c}
\theta \\
\beta
\end{array}\right)\left(\begin{array}{c}
\beta+p \\
p
\end{array}\right)\left(\begin{array}{c}
\theta-\beta+p \\
p
\end{array}\right)\right]^{\frac{1}{2}} .
$$


The operations on multi-indices are defined as

$$
\begin{aligned}
& \alpha=\beta \Leftrightarrow \alpha_{i}=\beta_{i} \forall i, \\
& \alpha \leqslant \beta \Leftrightarrow \alpha_{i} \leqslant \beta_{i} \forall i,
\end{aligned}
$$

and

$$
\alpha !=\prod_{i} \alpha_{i} !
$$

A.1. Finite truncation of the Wiener Chaos expansion and associated problems. We note that (2.4) is a double infinite expansion; this becomes clear when expressing the Wick polynomials in the expansion through the Hermite polynomials:

$$
u(x, t, \xi)=\sum_{\alpha \in \mathcal{J}} u_{\alpha}(x, t) T_{\alpha}(\xi)=\sum_{\alpha \in \mathcal{J}} u_{\alpha}(x, t) \prod_{i=1}^{\infty} H_{\alpha_{i}}\left(\xi_{i}\right) .
$$

Therefore in applications a 'double' truncation is performed [12]. Suppose we want to keep only $K$ random Gaussian variables $\xi_{i}$ and the Wick polynomials up to order $N$ in the approximation. Define the truncated index set

$$
\mathcal{J}_{K, N}=\left\{\alpha=\left(\alpha_{i}, i \geqslant 1 \mid \alpha_{i} \in\{0,1,2, \ldots, K\}\right),|\alpha|=\sum_{i=1}^{K} \alpha_{i} \leqslant N\right\} .
$$

Then, the truncated PCE has the form

$$
u_{K, N}(x, t, \xi)=\sum_{\alpha \in \mathcal{J}_{K, N}} u_{\alpha}(x, t) T_{\alpha}=\sum_{|\alpha| \leqslant N} u_{\alpha}(x, t) \prod_{i=1}^{K} H_{\alpha_{i}}\left(\xi_{i}\right) .
$$

The resulting approximation has altogether $\sum_{n=0}^{N}\left(\begin{array}{c}K+n-1 \\ n\end{array}\right)$ terms.

A.2. Spectral representation of the white noise. Since we are considering SDE's here we need a representation of the white noise that is compatible with the PC framework. We follow here the approach of [19, 20] and [12].

Consider any $T>0$ and orthonormal basis $\left\{m_{i}(s), i=1,2, \ldots\right\}$ in $L^{2}([0, T])$, for example the trigonometric functions

$$
m_{1}(s)=\frac{1}{\sqrt{T}}, \quad \text { and } \quad m_{i}(s)=\sqrt{\frac{2}{T}} \cos \left(\frac{(i-1) \pi s}{T}\right), \text { for } i=2,3, \ldots
$$

It is easy to see that the random variables defined by

$$
\xi_{i}=\int_{0}^{T} m_{i}(s) \mathrm{d} W(s), \quad i=1,2, \ldots
$$

are i.i.d. standard Gaussian random variables, $\xi_{i} \sim \mathcal{N}(0,1)$, so that the Wiener process can be represented by the following Fourier-Hermite expansion [12, 19, 20]:

$$
W(s)=\sum_{i=1}^{\infty} \xi_{i} \int_{0}^{s} m_{i}(\tau) \mathrm{d} W(\tau)
$$


The expansion (A.18) converges in the mean-square sense

$$
\left\langle\left[W(s)-\sum_{i=1}^{N} \xi_{i} \int_{0}^{s} m_{i}(\tau) \mathrm{d} W(\tau)\right]^{2}\right\rangle \underset{N \rightarrow \infty}{\longrightarrow} 0 .
$$

A.3. Truncated equations for the PCE coefficients of the test model. We assume here without a proof that solutions of (1.1) are second-order stable (methods similar to those used in [2] could be used to prove the second order stability of (1.1) when $a_{\gamma}$; otherwise careful Monte Carlo estimates are sufficient for our purposes). By the Cameron-Martin Theorem (see [4] and Appendix A) these solutions admit the PCE expansion (2.4). Substitution of (A.15) into (1.1) and utilizing the orthonormality of the Wick polynomials $T_{\alpha}$, together with the identity

$$
u \gamma=\sum_{\alpha \in \mathcal{J}}\left(\sum_{p \in \mathcal{J}} \sum_{0 \leqslant \beta \leqslant \alpha} C(\alpha, \beta, p) u_{\alpha-\beta+p} \gamma_{\beta+p}\right) T_{\alpha},
$$

due to Theorem 2 above (see also $[27,19,12,20]$ ), the truncated equations with $\alpha \in \mathcal{J}_{K, N}$ for the PCE coefficients of (1.1) are given by

$$
\begin{aligned}
& \text { a) } \dot{u}_{\alpha}=-\hat{\gamma} u_{\alpha}-a_{u} \sum_{p \in \mathcal{J}_{K, N}} \sum_{0 \leqslant \beta \leqslant \alpha} C(\alpha, \beta, p) u_{\alpha-\beta+p} \gamma_{\beta+p}+f(t) \delta_{\alpha \mathbf{0}}+\sigma_{u} \sum_{i=1} I_{\left\{\alpha_{i}=\delta_{i j}\right\}} m_{i}(t) \text {, } \\
& \text { b) } \dot{\gamma}_{\alpha}=-d_{\gamma} \gamma_{\alpha}-a_{\gamma} \sum_{p \in \mathcal{J}_{K, N}} \sum_{0 \leqslant \beta \leqslant \alpha} C(\alpha, \beta, p) u_{\alpha-\beta+p} \gamma_{\beta+p}+\sigma_{\gamma} \sum_{i=1} I_{\left\{\alpha_{i}=\delta_{i j}\right\}} m_{i}(t) \text {, }
\end{aligned}
$$

where, additionally, $u_{\alpha}=0$ for $\alpha \notin \mathcal{J}_{K, N}$.

Appendix B. Solutions of the Fokker-Planck equation for the test system (1.1) with intermittency via eigenfunction expansions. Below we complement the presentation of Section 2.2 with some technical details and derivations. Discussion of mathematical reasons for the limitations of the truncated Gram-Charlier expansions for UQ in systems with intermittency is presented in Section B.3 and Section B.5.

Consider first two Fokker-Planck equations associated with uncoupled scalar Ornstein-Uhlenbeck processes

$$
\begin{aligned}
& \partial_{t} p(u, t)=\hat{\gamma} \partial_{u}[u p(u, t)]+\frac{1}{2} \sigma_{u}^{2} \partial_{u}^{2} p(u, t), \\
& \partial_{t} q(\gamma, t)=d_{\gamma} \partial_{\gamma}[\gamma q(\gamma, t)]+\frac{1}{2} \sigma_{\gamma}^{2} \partial_{\gamma}^{2} q(\gamma, t),
\end{aligned}
$$

which are obtained by neglecting the quadratic coupling term in (1.1). The stationary solutions of (B.1) and (B.2) are the eigenfunctions of the Fokker-Planck operator with the eigenvalue $\lambda_{0}=0$; these solutions satisfy

$$
\hat{\gamma} \partial_{u}\left[u p_{e q}(u)\right]+\frac{1}{2} \sigma_{u}^{2} \partial_{u}^{2} p_{e q}(u)=0, \quad d_{\gamma} \partial_{\gamma}\left[\gamma q_{e q}(\gamma)\right]+\frac{1}{2} \sigma_{\gamma}^{2} \partial_{\gamma}^{2} q_{e q}(\gamma)=0,
$$

and are given by

$$
p_{e q}(u)=\left(\frac{\hat{\gamma}}{\pi \sigma_{u}^{2}}\right)^{1 / 2} \exp \left(-\frac{\hat{\gamma}}{\sigma_{u}^{2}} u^{2}\right)
$$




$$
q_{e q}(\gamma)=\left(\frac{d_{\gamma}}{\pi \sigma_{\gamma}^{2}}\right)^{1 / 2} \exp \left(-\frac{d_{\gamma}}{\sigma_{\gamma}^{2}} \gamma^{2}\right)
$$

We now rescale the variables $u$ and $\gamma$ using

$$
u=\mathcal{U}^{-1} \xi, \quad \mathcal{U}=\sqrt{\frac{\hat{\gamma}}{\sigma_{u}^{2}}} \quad \text { and } \quad \gamma=\mathcal{G}^{-1} \xi, \quad \mathcal{G}=\sqrt{\frac{d_{\gamma}}{\sigma_{\gamma}^{2}}}
$$

which transforms (B.1) and (B.2) to

$$
\begin{aligned}
& \partial_{t} p(\xi, t)=\hat{\gamma}\left(\partial_{\xi}[\xi p(\xi, t)]+\frac{1}{2} \partial_{\xi}^{2} p(\xi, t)\right) \equiv \hat{\gamma} \mathcal{L}_{F P} p(\xi, t), \\
& \partial_{t} q(\xi, t)=d_{\gamma}\left(\partial_{\xi}[\xi q(\xi, t)]+\frac{1}{2} \partial_{\xi}^{2} q(\xi, t)\right) \equiv d_{\gamma} \mathcal{L}_{F P} q(\xi, t),
\end{aligned}
$$

where the Fokker-Planck operator, $\mathcal{L}_{F P}$, in the rescaled variables, is the same in both cases. Furthermore, based on (B.4)-(B.6), the eigenfunction of (B.7), (B.8) corresponding to $\lambda_{0}=0$ can be written in both cases as

$$
\varphi_{0}(\xi)=N_{0} e^{-\xi^{2}}, \quad \mathcal{L}_{F P} \varphi_{0}=0,
$$

where $N_{0} \neq 0$ is a normalizing constant to be specified later.

Next, introduce an operator

$$
\tilde{\mathcal{L}} g(\xi) \equiv e^{\xi^{2} / 2} \mathcal{L}_{F P}\left(e^{-\xi^{2} / 2} g(\xi)\right),
$$

where $g \in C^{2}$ which can be easily evaluated as

$$
\tilde{\mathcal{L}} g \equiv \frac{1}{2}\left(\partial_{\xi}^{2} g+\left(1-\xi^{2}\right) g\right)
$$

Two properties of $\tilde{\mathcal{L}}$ are important for our considerations:

(i) $\tilde{\mathcal{L}}$ is self-adjoint,

(ii) $\tilde{\mathcal{L}}$ and $\mathcal{L}_{F P}$ have the same eigenvalues $\lambda_{n}$. Furthermore, if $\varphi_{n}$ is an eigenfunction of $\mathcal{L}_{F P}$, then

$$
\psi_{n}(\xi)=e^{\xi^{2} / 2} \varphi_{n}(\xi), \quad \psi_{0}(\xi)=N_{0} e^{-\xi^{2} / 2},
$$

is an eigenfunction of $\tilde{\mathcal{L}}$, i.e.,

$$
\tilde{\mathcal{L}} \psi_{n}=-\lambda_{n} \psi_{n} \quad \Leftrightarrow \quad \mathcal{L}_{F P} \varphi_{n}=-\lambda_{n} \varphi_{n}
$$

The proofs of (i) and (ii) are straightforward

Proof of (i): The operator $\tilde{\mathcal{L}}$ is a sum of two symmetric operators with respect to the canonical inner product in the Hilbert space. Thus, $\tilde{\mathcal{L}}$ is symmetric, i.e.,

$$
\int g \tilde{\mathcal{L}} h=\int h \tilde{\mathcal{L}} g .
$$

Hence, it is self-adjoint. In detail, for any $g, h \in C^{2}$ and vanishing at infinity

$$
\begin{aligned}
\int g \tilde{\mathcal{L}} h & =\int g\left(\partial_{\xi}^{2} h+\left(1-\xi^{2}\right) h\right) \mathrm{d} \xi=\int\left(g \partial_{\xi}^{2} h+\left(1-\xi^{2}\right) g h\right) \mathrm{d} \xi \\
& =\int\left(-\partial_{\xi} g \partial_{\xi} h+\left(1-\xi^{2}\right) g h\right) \mathrm{d} \xi=\int\left(h \partial_{\xi}^{2} g+\left(1-\xi^{2}\right) g h\right) \mathrm{d} \xi=\int h \tilde{\mathcal{L}} g
\end{aligned}
$$

Proof of (ii): This property follows immediately by a direct application of $\tilde{\mathcal{L}}$ to $\psi_{n}=e^{\xi^{2} / 2} \varphi_{n} ;$ namely

$$
\tilde{\mathcal{L}} \psi_{n}=e^{\xi^{2} / 2} \mathcal{L}_{F P}\left(e^{-\xi^{2} / 2} \psi_{n}\right)=e^{\xi^{2} / 2} \mathcal{L}_{F P}\left(\varphi_{n}\right)=-\lambda_{n} e^{\xi^{2} / 2} \varphi_{n}=-\lambda_{n} \psi_{n}
$$


B.1. Ladder operators associated with $\tilde{\mathcal{L}}$. We introduce here the ladder operators associated with $\tilde{\mathcal{L}}$ :

- the raising operator $b^{+}$:

$$
b^{+}=\frac{1}{\sqrt{2}}\left(-\partial_{\xi}+\xi\right)
$$

- and the lowering operator $b^{-}$:

$$
b^{-}=\frac{1}{\sqrt{2}}\left(\partial_{\xi}+\xi\right)
$$

with the following properties:

$$
\left[\tilde{\mathcal{L}}, b^{+}\right]=-b^{+}, \quad\left[\tilde{\mathcal{L}}, b^{-}\right]=b^{-}, \quad\left[b^{-}, b^{+}\right]=1,
$$

where the commutator of two operators $[X, Y]$ is defined in the standard way as $[X, Y]=X Y-Y X$.

Notice first that the eigenvalues of $\tilde{\mathcal{L}}$ and $\mathcal{L}_{F P}$ are given by $\lambda_{n}=n$. Next, consider an eigenfunction $\psi_{n}$ of $\tilde{\mathcal{L}}$. It is straightforward to see that the action of the raising operator on the eigenfunction $\psi_{n}$ yields the eigenfunction $\psi_{n+1}$; namely

$$
\tilde{\mathcal{L}} b^{+} \psi_{n}=\left(b^{+} \tilde{\mathcal{L}}+\left[\tilde{\mathcal{L}}, b^{+}\right]\right) \psi_{n}=\left(b^{+} \tilde{\mathcal{L}}-2 b^{+}\right) \psi_{n}=-(n+1) b^{+} \psi_{n}
$$

Analogously, the lowering operator $b^{-}$acts to produce the eigenfunction $\psi_{n-1}$ from $\psi_{n}$ :

$$
\tilde{\mathcal{L}} b^{-} \psi_{n}=\left(b^{-} \tilde{\mathcal{L}}+\left[\tilde{\mathcal{L}}, b^{-}\right]\right) \psi_{n}=\left(b^{-} \tilde{\mathcal{L}}+2 b^{-}\right) \psi_{n}=-(n-1) b^{-} \psi_{n} .
$$

Note, in particular, that

$$
b^{-} \psi_{0}=\frac{N_{0}}{\sqrt{2}}\left(\partial_{\xi}+\xi\right) e^{-\xi^{2} / 2}=0 .
$$

We summarize the properties of the ladder operators as

$$
b^{+} \psi_{n}=\psi_{n+1}, \quad b^{-} \psi_{n}=\sqrt{2} n \psi_{n-1}, \quad b^{-} \psi_{0}=0 .
$$

B.2. Eigenfunctions of $\tilde{\mathcal{L}}$ and $\mathcal{L}_{F P}$. We can easily obtain the eigenfunctions of $\tilde{\mathcal{L}}$ and $\mathcal{L}_{F P}$ with the help of the raising operator $b^{+}$and the property (ii).

The orthonormal basis of $\tilde{\mathcal{L}}$ is given by

$$
\begin{aligned}
& \psi_{0}=\frac{1}{\pi^{1 / 4}} e^{-\xi^{2} / 2} \\
& \psi_{n}=\frac{\left(b^{+}\right)^{n}}{\sqrt{2^{n} n !}} \psi_{0}=\frac{1}{\pi^{1 / 4} \sqrt{2^{n} n !}} H_{n}(\xi) e^{-\xi^{2} / 2} \\
& \int_{-\infty}^{\infty} \psi_{n}(\xi) \psi_{m}(\xi) \mathrm{d} \xi=\delta_{n, m},
\end{aligned}
$$

where $H_{n}$ is the $n$-th Hermite polynomial satisfying

$$
H_{n}^{\prime \prime}-2 \xi H_{n}^{\prime}+2 n H_{n}=0 .
$$




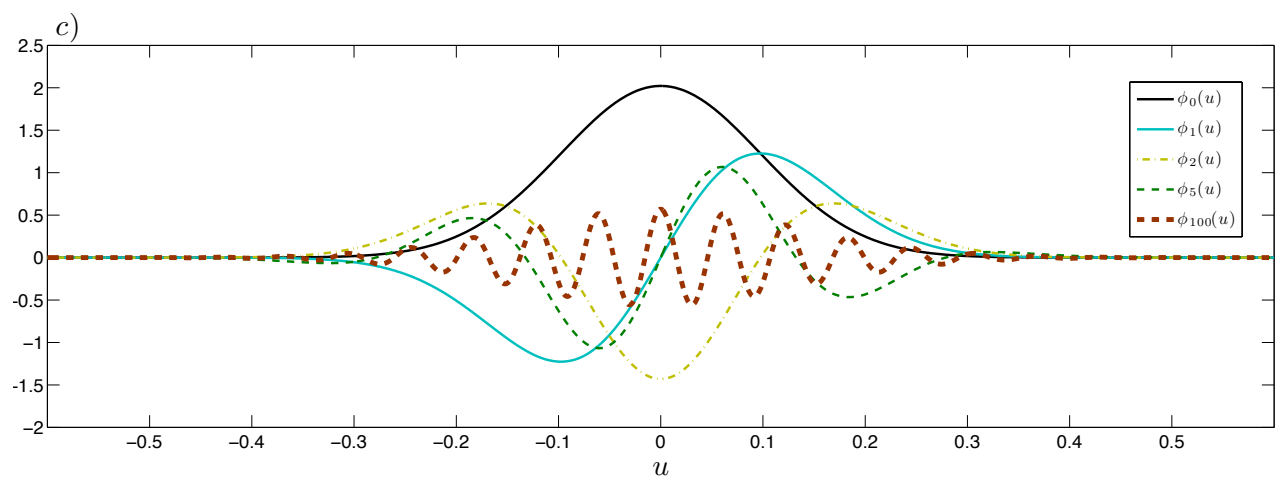

FIG. B.1. Illustration of the eigenfunctions of the Fokker-Planck operators $\mathcal{L}_{F P}^{u}$ and $\mathcal{L}_{F P}^{\gamma}$ defined in (B.7) and (B.8).

The action of the ladder operators on the orthonormal set yields

$$
b^{+} \psi_{n}=(n+1)^{1 / 2} \psi_{n+1}, \quad b^{-} \psi_{n}=n^{1 / 2} \psi_{n-1}, \quad b^{-} \psi_{0}=0 .
$$

Using the fact that $\psi_{n}=e^{\xi^{2} / 2} \varphi_{n}$ (cf. Property (ii)) the orthonormal set for $\mathcal{L}_{F P}$ with weight function $\omega(\xi)=e^{\xi^{2}}$ is

$$
\begin{aligned}
& \varphi_{0}=\frac{1}{\pi^{1 / 4}} e^{-\xi^{2}} \\
& \varphi_{n}=\frac{\left(b^{+}\right)^{n}}{\sqrt{2^{n} n !}} \psi_{0}=\frac{1}{\pi^{1 / 4} \sqrt{2^{n} n !}} H_{n}(\xi) e^{-\xi^{2}} \\
& \int_{-\infty}^{\infty} \varphi_{n}(\xi) \varphi_{m}(\xi) \omega(\xi) \mathrm{d} \xi=\delta_{n, m} .
\end{aligned}
$$

In the original variables the eigenvalues of $\mathcal{L}_{F P}^{u}$ and $\mathcal{L}_{F P}^{\gamma}$ are, respectively, $\lambda_{k}^{u}=-\hat{\gamma} k$ and $\lambda_{l}^{u}=-d_{\gamma} l$, with the eigenfunctions

$$
\begin{aligned}
& \phi_{k}(u)=\mathcal{U}^{1 / 2} \varphi_{k}(\mathcal{U} u), \quad \mathcal{U}=\sqrt{\frac{\hat{\gamma}}{\sigma_{u}^{2}}}, \\
& \phi_{l}(\gamma)=\mathcal{G}^{1 / 2} \varphi_{l}(\mathcal{G} \gamma), \quad \mathcal{G}=\sqrt{\frac{d_{\gamma}}{\sigma_{\gamma}^{2}}} .
\end{aligned}
$$

That is

$$
\phi_{k}(u)=\left(\frac{\hat{\gamma}}{\pi \sigma_{u}^{2}}\right)^{1 / 4} \frac{1}{\sqrt{2^{k} k !}} H_{k}\left(\sqrt{\frac{\hat{\gamma}}{\sigma_{u}^{2}}} u\right) e^{-u^{2} \hat{\gamma} / \sigma_{u}^{2}}
$$

and

$$
\phi_{l}(\gamma)=\left(\frac{d_{\gamma}}{\pi \sigma_{\gamma}^{2}}\right)^{1 / 4} \frac{1}{\sqrt{2^{l} l !}} H_{l}\left(\sqrt{\frac{d_{\gamma}}{\sigma_{\gamma}^{2}}} \gamma\right) e^{-\gamma^{2} d_{\gamma} / \sigma_{\gamma}^{2}}
$$


B.3. Equations for the coefficients in the Gram-Charlier expansion.

Below we derive a linear system of equations for the coefficients $C_{k, l}$ in the eigenfunction expansion (2.16); the finitely truncated system for such coefficients is shown here to be

$$
\begin{aligned}
\frac{\mathrm{d} C_{k, l}^{M}}{\mathrm{~d} t}= & -\lambda_{k, l} C_{k, l}^{M} \\
& -\alpha_{k}\left((l+1)^{1 / 2} C_{k, l+1}^{M}+l^{1 / 2} C_{k, l-1}^{M}\right)-\beta_{k}\left((l+1)^{1 / 2} C_{k-2, l+1}^{M}+l^{1 / 2} C_{k-2, l-1}^{M}\right) \\
& -\tilde{\alpha}_{l}\left((k+1)^{1 / 2} C_{k+1, l}^{M}+k^{1 / 2} C_{k-1, l}^{M}\right)-\tilde{\beta}_{l}\left((k+1)^{1 / 2} C_{k+1, l-2}^{M}+k^{1 / 2} C_{k-1, l-2}^{M}\right) \\
& +\kappa_{k} f(t) C_{k-1, l}^{M}, \quad \text { for } \mathrm{k} \in[0,1, \ldots, \mathrm{K}], 1 \in[0,1, \ldots, \mathrm{L}],
\end{aligned}
$$

and

$$
C_{k, l}^{M}=0, \quad \text { for } \quad k \notin[0,1, \ldots, K], l \notin[0,1, \ldots, L] .
$$

Detailed derivation of the above systems is as follows:

The Fokker-Planck for the system (1.1) can be written as

$$
\partial_{t} p(u, \gamma, t)=\mathcal{L}_{F P}^{u} p+\mathcal{L}_{F P}^{\gamma} p+\partial_{u}\left[\left(a_{u} \gamma u-f(t)\right) p\right]+a_{\gamma} u \partial_{\gamma}[\gamma p],
$$

where the two operators $\mathcal{L}_{F P}^{u}$ and $\mathcal{L}_{F P}^{\gamma}$ associated with the decoupled system (i.e., when $a_{u}=a_{\gamma}=0$ ) were defined in (B.7) and (B.8). Assume now the following expansion of the joint density $p(u, \gamma, t)$ in eigenfunctions of the self-adjoint operator $\mathcal{L}_{F P}^{u}+\mathcal{L}_{F P}^{\gamma}:$

$$
p(u, \gamma, t)=\sum_{k, l=1}^{\infty} C_{k, l}(t) \phi_{k}(u) \phi_{l}(\gamma) .
$$

Substitution of (B.39) into (B.38) leads to

$$
\begin{aligned}
\sum_{k, l} \frac{\mathrm{d} C_{k, l}(t)}{\mathrm{d} t} \phi_{k}(u) \phi_{l}(\gamma)=-\sum_{k, l} & \lambda_{k, l} C_{k, l}(t) \phi_{k}(u) \phi_{l}(\gamma) \\
& +a_{u} \sum_{k, l} C_{k, l}(t) \gamma \phi_{l}(\gamma) \phi_{k}(u) \\
& +a_{\gamma} \sum_{k, l} C_{k, l}(t) \phi_{l}(\gamma) u \phi_{k}(u) \\
& +a_{u} \sum_{k, l} C_{k, l}(t) \gamma \phi_{l}(\gamma) u \partial_{u}\left[\phi_{k}(u)\right] \\
& +a_{\gamma} \sum_{k, l} C_{k, l}(t) u \phi_{k}(u) \gamma \partial_{\gamma}\left[\phi_{l}(\gamma)\right] \\
& -\sum_{k, l} C_{k, l}(t) f(t) \phi_{l}(\gamma) \partial_{u}\left[\phi_{k}(u)\right],
\end{aligned}
$$

where

$$
\lambda_{k, l}=\hat{\gamma} k+d_{\gamma} l
$$


is the eigenvalue of the decoupled operator $\mathcal{L}_{F P}^{u}+\mathcal{L}_{F P}^{\gamma}$. The evolution of the coefficient $C_{k, l}$ can be obtained by exploiting the orthonormality of the eigenfunctions with appropriate weights $\varpi(u), \varpi(\gamma)$ (see (B.29)-(B.31)). Consequently, we have the initial conditions $C_{k, l}^{0}$ determined by the initial distribution $p(u, \gamma, t=0)$. Clearly, in order to compute the appropriate Galerkin projections, one needs to evaluate the following integrals:

$$
\begin{array}{cl}
\text { (I) } & \int_{-\infty}^{\infty} \gamma \phi_{l}(\gamma) \phi_{l^{\prime}}(\gamma) \varpi(\gamma) \mathrm{d} \gamma, \\
(I I) \quad & \int_{-\infty}^{\infty} u \phi_{k}(u) \phi_{k^{\prime}}(u) \varpi(u) \mathrm{d} u, \\
(I I I) \quad & \int_{-\infty}^{\infty} u \partial_{u}\left[\phi_{k}(u)\right] \phi_{k^{\prime}}(u) \varpi(u) \mathrm{d} u \\
(I V) \quad & \int_{-\infty}^{\infty} \gamma \partial_{\gamma}\left[\phi_{l}(\gamma)\right] \phi_{l^{\prime}}(\gamma) \varpi(\gamma) \mathrm{d} \gamma, \\
& \quad \int_{-\infty}^{\infty} \partial_{u}\left[\phi_{k}(u)\right] \phi_{k^{\prime}}(u) \varpi(u) \mathrm{d} u .
\end{array}
$$

where (I), (II) have the same structure and, similarly, so do (III), (IV). Recall that

$$
\begin{aligned}
& \phi_{k}(u)=\mathcal{U}^{1 / 2} \varphi_{k}(\mathcal{U} u), \quad \mathcal{U}=\sqrt{\frac{\hat{\gamma}}{\sigma_{u}^{2}}} \\
& \phi_{l}(\gamma)=\mathcal{G}^{1 / 2} \varphi_{l}(\mathcal{G} \gamma), \quad \mathcal{G}=\sqrt{\frac{d_{\gamma}}{\sigma_{\gamma}^{2}}} .
\end{aligned}
$$

The above integrals can be evaluated as follows:

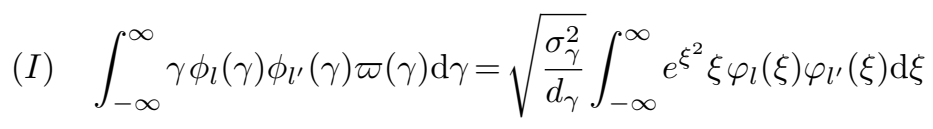

$$
\begin{aligned}
& =\sqrt{\frac{\sigma_{\gamma}^{2}}{d_{\gamma}}} \int_{-\infty}^{\infty} \xi \psi_{l}(\xi) \psi_{l^{\prime}}(\xi) \mathrm{d} \xi \\
& =\sqrt{\frac{\sigma_{\gamma}^{2}}{2 d_{\gamma}}} \int_{-\infty}^{\infty}\left(b^{+}+b^{-}\right) \psi_{l}(\xi) \psi_{l^{\prime}}(\xi) \mathrm{d} \xi \\
& =\sqrt{\frac{\sigma_{\gamma}^{2}}{2 d_{\gamma}}} \int_{-\infty}^{\infty}\left((l+1)^{1 / 2} \psi_{l+1}(\xi) \psi_{l^{\prime}}(\xi)+l^{1 / 2} \psi_{l-1}(\xi) \psi_{l^{\prime}}(\xi)\right) \mathrm{d} \xi \\
& =\sqrt{\frac{\sigma_{\gamma}^{2}}{2 d_{\gamma}}}\left((l+1)^{1 / 2} \delta_{l+1-l^{\prime}}+l^{1 / 2} \delta_{l-1-l^{\prime}}\right) .
\end{aligned}
$$

and

(II) $\int_{-\infty}^{\infty} u \phi_{k}(u) \phi_{k^{\prime}}(u) \varpi(u) \mathrm{d} u=\sqrt{\frac{\sigma_{u}^{2}}{2 \hat{\gamma}}}\left((k+1)^{1 / 2} \delta_{k+1-k^{\prime}}+k^{1 / 2} \delta_{k-1-k^{\prime}}\right)$, 
where we used $\xi=\left(b^{+}+b^{-}\right) / \sqrt{2}$ and relations (B.28). The integrals (III) and (IV) are computed in a similar fashion leading to

$$
\begin{aligned}
(I I I) & \int_{-\infty}^{\infty} u \partial_{u}\left[\phi_{k}(u)\right] \phi_{k^{\prime}}(u) \varpi(u) \mathrm{d} u=\int_{-\infty}^{\infty} e^{\xi^{2}} \xi \partial_{\xi}\left[\varphi_{k}(\xi)\right] \varphi_{k^{\prime}}(\xi) \mathrm{d} \xi \\
= & \int_{-\infty}^{\infty} \xi e^{\xi^{2} / 2} \partial_{\xi}\left[e^{-\xi^{2} / 2} \psi_{k}(\xi)\right] \psi_{k^{\prime}}(\xi) \mathrm{d} \xi=\int_{-\infty}^{\infty} \xi\left(\partial_{\xi} \psi_{k}(\xi)-\xi \psi_{k}(\xi)\right) \psi_{k^{\prime}}(\xi) \mathrm{d} \xi \\
= & -\sqrt{2} \int_{-\infty}^{\infty} \xi b^{+} \psi_{k}(\xi) \psi_{k^{\prime}}(\xi) \mathrm{d} \xi=-\sqrt{2} \int_{-\infty}^{\infty} \xi(k+1)^{1 / 2} \psi_{k+1}(\xi) \psi_{k^{\prime}}(\xi) \mathrm{d} \xi \\
= & -(k+1)^{1 / 2} \int_{-\infty}^{\infty}\left(b^{+}+b^{-}\right) \psi_{k+1}(\xi) \psi_{k^{\prime}}(\xi) \mathrm{d} \xi \\
= & -(k+1)^{1 / 2 f} \int_{-\infty}^{\infty}\left((k+2)^{1 / 2} \psi_{k+2}(\xi) \psi_{k^{\prime}}(\xi)+(k+1)^{1 / 2} \psi_{k}(\xi) \psi_{k^{\prime}}(\xi)\right) \mathrm{d} \xi \\
= & -(k+1)^{1 / 2}\left((k+2)^{1 / 2} \delta_{k+2-k^{\prime}}+(k+1)^{1 / 2} \delta_{k-k^{\prime}}\right)
\end{aligned}
$$

and

$$
\int_{-\infty}^{\infty} \gamma \partial_{\gamma}\left[\phi_{l}(\gamma)\right] \phi_{l^{\prime}}(\gamma) \varpi(\gamma) \mathrm{d} \gamma=-(l+1)^{1 / 2}\left((l+2)^{1 / 2} \delta_{l+2-l^{\prime}}+(l+1)^{1 / 2} \delta_{l-l^{\prime}}\right) .
$$

Finally, the integral (V) is evaluated as follows

$$
\begin{aligned}
& \int_{-\infty}^{\infty} \partial_{u}\left[\phi_{k}(u)\right] \phi_{k^{\prime}}(u) \varpi(u) \mathrm{d} u=\sqrt{\frac{\hat{\gamma}}{\sigma_{u}^{2}}} \int_{-\infty}^{\infty} e^{\xi^{2}} \partial_{\xi}\left[\varphi_{k}(\xi)\right] \varphi_{k^{\prime}}(\xi) \mathrm{d} \xi \\
= & \int_{-\infty}^{\infty} e^{\xi^{2} / 2} \partial_{\xi}\left[e^{-\xi^{2} / 2} \psi_{k}(\xi)\right] \psi_{k^{\prime}}(\xi) \mathrm{d} \xi=\int_{-\infty}^{\infty}\left(\partial_{\xi} \psi_{k}(\xi)-\xi \psi_{k}(\xi)\right) \psi_{k^{\prime}}(\xi) \mathrm{d} \xi \\
= & -\sqrt{2} \int_{-\infty}^{\infty} b^{+} \psi_{k}(\xi) \psi_{k^{\prime}}(\xi) \mathrm{d} \xi=-\sqrt{2} \int_{-\infty}^{\infty}(k+1)^{1 / 2} \psi_{k+1}(\xi) \psi_{k^{\prime}}(\xi) \mathrm{d} \xi \\
= & -\sqrt{\frac{2 \hat{\gamma}}{\sigma_{u}^{2}}}(k+1)^{1 / 2} \delta_{k+1-k^{\prime}} .
\end{aligned}
$$

Upon substitution of the expressions (I)-(V) into (B.40), the linear infinite system of ODEs for the coefficients $C_{k, l}$ can be written as

$$
\begin{aligned}
\frac{\mathrm{d} C_{k, l}}{\mathrm{~d} t}= & -\lambda_{k, l} C_{k, l} \\
& -\alpha_{k}\left((l+1)^{1 / 2} C_{k, l+1}+l^{1 / 2} C_{k, l-1}\right)-\beta_{k}\left((l+1)^{1 / 2} C_{k-2, l+1}+l^{1 / 2} C_{k-2, l-1}\right) \\
& -\tilde{\alpha}_{l}\left((k+1)^{1 / 2} C_{k+1, l}+k^{1 / 2} C_{k-1, l}\right)-\tilde{\beta}_{l}\left((k+1)^{1 / 2} C_{k+1, l-2}+k^{1 / 2} C_{k-1, l-2}\right) \\
& +\kappa_{k} f(t) C_{k-1, l},
\end{aligned}
$$

where

$$
\alpha_{k}=a_{u} \sqrt{\frac{\sigma_{\gamma}^{2}}{2 d_{\gamma}}} k, \quad \beta_{k}=a_{u} \sqrt{\frac{\sigma_{\gamma}^{2}}{2 d_{\gamma}}} k^{1 / 2}(k-1)^{1 / 2}, \quad \kappa_{k}=\sqrt{\frac{2 \hat{\gamma}}{\sigma_{u}^{2}}} k^{1 / 2},
$$




$$
\tilde{\alpha}_{l}=a_{\gamma} \sqrt{\frac{\sigma_{u}^{2}}{2 \hat{\gamma}}} l, \quad \tilde{\beta}_{l}=a_{\gamma} \sqrt{\frac{\sigma_{u}^{2}}{2 \hat{\gamma}}} l^{1 / 2}(l-1)^{1 / 2} .
$$
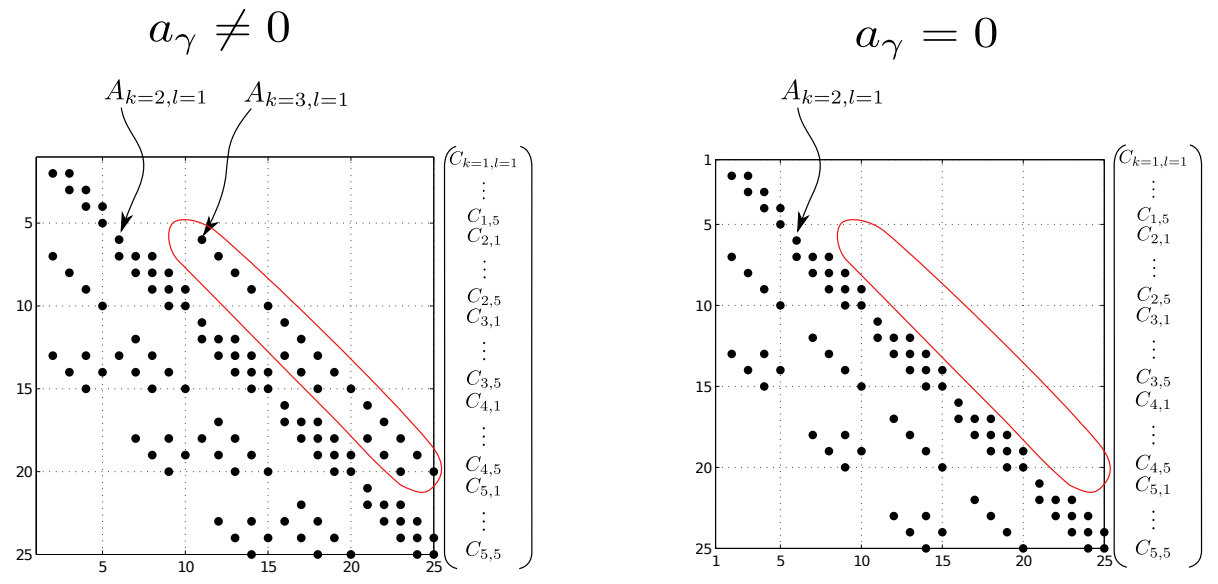

FIG. B.2. (Left) The general structure of the matrix $A_{\boldsymbol{M}}$ in (2.18) of the truncated system (B.36) with $K=L=5$ for the coefficients in the eigenfunction expansion of the probability density $p(u, \gamma, t)$ associated with (1.1). For $a_{\gamma}=0$ in the system (1.1) the matrix $A_{\boldsymbol{M}}$ is an upper Hessenberg matrix (i.e. lower triangular plus the first superdiagonal) which leads to a specific closedness property resulting in the evolution of $C_{k, l}$ depending only on $C_{k^{\prime}, l^{\prime}}$ with $k^{\prime} \leqslant k, l^{\prime}=0, \ldots, L$.

Any practical application of the expansion (B.39) requires a finite-dimensional truncation which leads to

$$
p^{\boldsymbol{M}}(u, \gamma, t)=\sum_{\substack{0 \leqslant k \leqslant K, 0 \leqslant l \leqslant L}} C_{k, l}^{\boldsymbol{M}} \phi_{k}(u) \phi_{l}(\gamma), \quad \boldsymbol{M}=(K, L),
$$

so that the coefficients, $C_{k, l}^{M}$, in the truncated expansion (2.17) satisfy a linear system

$$
\frac{\mathrm{d} \boldsymbol{C}^{\boldsymbol{M}}}{\mathrm{d} t}=A_{\boldsymbol{M}}(t) \boldsymbol{C}^{\boldsymbol{M}},
$$

where truncated vector of coefficients is $\boldsymbol{C}^{\boldsymbol{M}}=\left(C_{1,(1, \ldots, L)}, C_{2,(1, \ldots, L)}, \ldots, C_{K,(1, \ldots, L)}\right)^{T}$; the structure of the resulting matrix is shown in figure B.2. Alternatively, the truncated system can be written as

$$
\begin{aligned}
\frac{\mathrm{d} C_{k, l}^{M}}{\mathrm{~d} t}= & -\lambda_{k, l} C_{k, l}^{M} \\
& -\alpha_{k}\left((l+1)^{1 / 2} C_{k, l+1}^{M}+l^{1 / 2} C_{k, l-1}^{M}\right)-\beta_{k}\left((l+1)^{1 / 2} C_{k-2, l+1}^{M}+l^{1 / 2} C_{k-2, l-1}^{M}\right) \\
& -\tilde{\alpha}_{l}\left((k+1)^{1 / 2} C_{k+1, l}^{M}+k^{1 / 2} C_{k-1, l}^{M}\right)-\tilde{\beta}_{l}\left((k+1)^{1 / 2} C_{k+1, l-2}^{M}+k^{1 / 2} C_{k-1, l-2}^{M}\right) \\
& +\kappa_{k} f(t) C_{k-1, l}^{M}, \quad \text { for } \quad k \in[0,1, \ldots, K], l \in[0,1, \ldots, L],
\end{aligned}
$$


and

$$
C_{k, l}^{M}=0, \quad \text { for } \quad k \notin[0,1, \ldots, K], l \notin[0,1, \ldots, L] .
$$
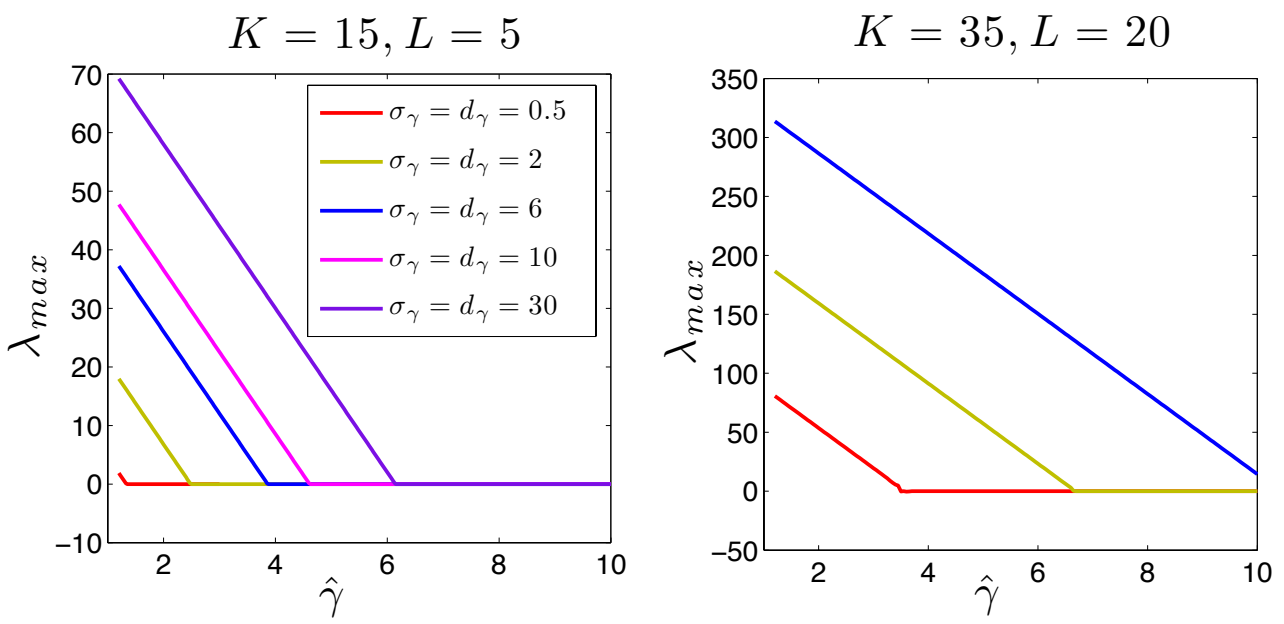

FIG. B.3. Erroneous instabilities in the truncated system for the coefficients in the GrCh expansion. The largest eigenvalue of the matrix of the truncated system (2.18) (see also figure B.2) as a function of the mean damping $\hat{\gamma}$ for different truncations and varied $\sigma_{\gamma}, d_{\gamma}$. The matrix of the truncated system loses positive semi-definiteness for sufficiently small $\hat{\gamma}$ (i.e. for sufficiently small numbers of finite moments in the equilibrium PDF of (1.1)). The above results were obtained with $a_{\gamma}=0, \sigma_{u}=0.5$ but there is little change for perturbations with $a_{\gamma} \ll 1$ in (1.1).

We note two important properties of the system (B.58)

(i) For $a_{\gamma}=0$ in (1.1) so that $\tilde{\alpha}_{l}=\tilde{\beta}_{l}=0$ for all $l$, the evolution of $C_{k, l}$ in $(2.18)$ involves only terms with $k^{\prime} \leqslant k$ :

$$
\frac{\mathrm{d}}{\mathrm{d} t} C_{k, l} \propto \sum_{k^{\prime} \leqslant k, l^{\prime}} C_{k^{\prime}, l^{\prime}}
$$

this property is due to properties of Hermite polynomials exploited in the evaluation of the integrals (I)-(V) above.

(ii) The truncation directly affects the entries multiplying $C_{k-2, L+1}^{\boldsymbol{M}}$ and $C_{k, L+1}^{\boldsymbol{M}}$, $k=0, \ldots, K$, and the removed coefficients grow with $L$.

ERroneous INSTABILITIES IN THE TRUNCATED SYSTEM DUE TO ONSET OF INTERMITTENCY

As discussed in Section 2.2, the GrChE method relies on computing the evolution of the time-dependent coefficients in the truncated spectral expansion of the probability density in eigenfunctions of the corresponding Fokker-Planck operator. An important drawback of the finite truncation, which is particularly problematic in systems with intermittency, is associated with erroneous exponential instabilities in the linear system for the expansion coefficients. These effects, which we illustrate in figure B.3, highlight the following facts: 
- For sufficiently weak mean damping $\hat{\gamma}$ (leading to increasingly intermittent dynamics) the truncated system (B.36) for the expansion coefficient has a positive eigenvalue $\lambda_{\max }>0$ which leads to erroneous exponential instability coupled with an onset of non-realizability of the GrChE approximations.

- The threshold $\hat{\gamma}_{\min }$ for the onset of such instabilities in the truncated system (B.36) increases with the order of truncation and as the damping fluctuations approach the white noise limit (i.e., increasingly large mean damping is needed to prevent onset of the instability).

It is also shown in figure 5.4 that even if the truncated system (B.36) is stable (i.e., $\lambda_{\max }=0$ ), the corresponding approximate equilibrium solutions are generally non-realizable as probability densities with spurious large amplitude components dominating the solutions. Moreover, for very low-order expansions (2.17) the truncated system (B.36) is stable and the approximate solutions are realizable PDFs, but they completely miss the intermittency in the true marginal PDF for $u$ satisfying (1.1).

B.4. Second order statistics of $u$ from the truncated system. Given the approximate density $p^{\boldsymbol{M}}(u, \gamma, t)$ it is possible to compute the approximate secondorder statistics of $u$ as

$$
\bar{u}^{\boldsymbol{M}}(t)=\iint u p^{\boldsymbol{M}}(u, \gamma, t) \mathrm{d} u \mathrm{~d} \gamma, \quad \operatorname{Var}\left(u^{\boldsymbol{M}}(t)\right)=\iint(u-\bar{u})^{2} p^{\boldsymbol{M}}(u, \gamma, t) \mathrm{d} u \mathrm{~d} \gamma .
$$

We note that the expressions in (B.61) can be simplified using the fact that if $f(x)$ is a polynomial of degree less than $n$, then

$$
\int_{-\infty}^{\infty} f(x) H_{m}(x) e^{-x^{2}} \mathrm{~d} x=0, \quad m>n .
$$

Therefore, we have

$$
\begin{aligned}
\bar{u}^{M}(t) & =\int_{-\infty}^{\infty} \varphi_{0}(\gamma) \mathrm{d} \gamma \sum_{\alpha=0,1} C_{\alpha, 0}(t) \int_{-\infty}^{\infty} u \varphi_{\alpha}(u) \mathrm{d} u \\
& =\left(\frac{\pi \sigma_{\gamma}^{2}}{d_{\gamma}}\right)^{1 / 4}\left(C_{0,0}(t) \int_{-\infty}^{\infty} u \varphi_{0}(u) \mathrm{d} u+C_{1,0}(t) \int_{-\infty}^{\infty} u \varphi_{1}(u) \mathrm{d} u\right) \\
& =\left(\frac{\sigma_{\gamma}^{2}}{d_{\gamma}}\right)^{1 / 4}\left(\frac{\sigma_{u}^{2}}{\hat{\gamma}}\right)^{3 / 4}\left(\frac{\pi}{2}\right)^{1 / 2} C_{1,0}(t) \\
\operatorname{Var}\left(u^{M}(t)\right) & =\int_{-\infty}^{\infty} \varphi_{0}(\gamma) \mathrm{d} \gamma \sum_{\alpha=0,1,2} C_{\alpha, 0}(t) \int_{-\infty}^{\infty}(u-\bar{u})^{2} \varphi_{\alpha}(u) \mathrm{d} u \\
& =\left(\frac{\pi \sigma_{\gamma}^{2}}{d_{\gamma}}\right)^{1 / 4}\left(\frac{\sigma_{u}^{2}}{\hat{\gamma}}\right)^{5 / 4} \frac{\pi^{1 / 4}}{2}\left(C_{0,0}(t)+\sqrt{2} C_{2,0}(t)\right) \\
& -\left(\frac{\sigma_{\gamma}^{2}}{d_{\gamma}}\right)^{1 / 2}\left(\frac{\sigma_{u}^{2}}{\hat{\gamma}}\right)^{3 / 2} \frac{\pi}{2} C_{1,0}^{2}(t)
\end{aligned}
$$

Important points to note regarding the above results are: 
(i) The second-order statistics are determined by the three coefficients $C_{0,0}, C_{1,0}$, and $\mathrm{C}_{2,0}$.

(ii) For $a_{\gamma}=0$ in (1.1), and due to the property (B.60) from the previous section, the evolution of the second-order statistics expressed through $C_{0,0}(t), C_{1,0}(t), C_{2,0}(t)$ depends only on terms $C_{0, l}, C_{1, l}, C_{2, l}$ with $l=0, \ldots, L$ (see figure B.2).

(ii) For $\quad a \neq 0$ the second-order statistics expressed through $C_{0,0}(t), C_{1,0}(t), C_{2,0}(t)$ are coupled to $C_{k, l}$ with $k>2$ (see figure B.2).

B.5. Class of degenerate two-dimensional systems preserving the closedness property of (1.1) with $a_{\gamma}=0$. We show here that any extension of the test model (1.1) with $a_{\gamma}=0$ to

$$
\begin{aligned}
& \text { a) } \mathrm{d} u(t)=(-(\hat{\gamma}+\gamma(t)) u(t)+f(t)) \mathrm{d} t+\sigma_{u} \mathrm{~d} W_{u}(t), \\
& \text { b) } \mathrm{d} \gamma(t)=-\left(d_{\gamma} \gamma+F(\gamma(t))\right) \mathrm{d} t+\sigma_{\gamma} \mathrm{d} W_{\gamma}(t),
\end{aligned}
$$

with $F(\gamma) \sim \mathcal{O}\left(\gamma^{2}\right)$ leads to a linear system for the $\mathrm{GrChE}$ coefficients $C_{k, l}$ with a similar lower-triangular structure as (2.18) for the system (1.1) shown in figure B.2b. Consequently, such systems will possess the same closedness property (B.60) as (1.1) with $a_{\gamma}=0$.

Consider the Fokker-Planck for the full system (B.65) given by

$$
\partial_{t} p(u, \gamma, t)=\frac{1}{2} \sigma_{u}^{2} \partial_{u}^{2} p+\hat{\gamma} \partial_{u}[u p]+\frac{1}{2} \sigma_{\gamma}^{2} \partial_{\gamma}^{2} p+d_{\gamma} \partial_{\gamma}[\gamma p]+\partial_{u}[(\gamma u-f(t)) p]+\partial_{\gamma}[F(\gamma) p],
$$

which can be written as

$$
\partial_{t} p(u, \gamma, t)=\mathcal{L}_{F P}^{u} p+\mathcal{L}_{F P}^{\gamma} p+\partial_{u}[(\gamma u-f(t)) p]+\partial_{\gamma}[F(\gamma) p],
$$

where

$$
\begin{aligned}
\mathcal{L}_{F P}^{u} p & =\frac{1}{2} \sigma_{u}^{2} \partial_{u}^{2} p+\hat{\gamma} \partial_{u}[u p], \\
\mathcal{L}_{F P}^{\gamma} p & =\frac{1}{2} \sigma_{\gamma}^{2} \partial_{\gamma}^{2} p+d_{\gamma} \partial_{\gamma}[\gamma p] .
\end{aligned}
$$

In the absence of the coupling term $\gamma u$ and $F(\gamma) \equiv 0$, the Fokker-Planck equation (B.38) can be separated using the following expansion:

$$
p(u, \gamma, t)=\sum_{k, l=1}^{\infty} C_{k, l}(t) \phi_{k}(u) \phi_{l}(\gamma),
$$

where $\phi_{k}(u), \phi_{l}(\gamma)$ are the same as before.

The evolution of the element $C_{k, l}$ can be obtained by exploiting the orthogonality of the eigenfunctions with appropriate weight functions $\varpi(u), \varpi(\gamma)$ (see (B.29)-(B.31))

$$
\begin{aligned}
\sum_{k, l} \frac{\mathrm{d} C_{k, l}(t)}{\mathrm{d} t} \phi_{k}(u) \phi_{l}(\gamma)=-\sum_{k, l} & \lambda_{k, l} C_{k, l}(t) \phi_{k}(u) \phi_{l}(\gamma)+\sum_{k, l} C_{k, l}(t) \gamma \phi_{l}(\gamma) \phi_{k}(u) \\
& +\sum_{k, l} C_{k, l}(t) \phi_{l}(\gamma) F^{\prime}(\gamma) \phi_{k}(u) \\
& +\sum_{k, l} C_{k, l}(t) \gamma \phi_{l}(\gamma) u \partial_{u}\left[\phi_{k}(u)\right]
\end{aligned}
$$




$$
\begin{aligned}
& +\sum_{k, l} C_{k, l}(t) \phi_{k}(u) F(\gamma) \partial_{\gamma}\left[\phi_{l}(\gamma)\right] \\
& -\sum_{k, l} C_{k, l}(t) f(t) \phi_{l}(\gamma) \partial_{u}\left[\phi_{k}(u)\right]
\end{aligned}
$$

Clearly, in order to compute the appropriate Galerkin projections, one needs to evaluate the following integrals:

$$
\begin{array}{cl}
\text { (I) } \quad & \int_{-\infty}^{\infty} \gamma \phi_{l}(\gamma) \phi_{l^{\prime}}(\gamma) \varpi(\gamma) \mathrm{d} \gamma, \\
(I I) \quad & \int_{-\infty}^{\infty} u \partial_{u}\left[\phi_{k}(u)\right] \phi_{k^{\prime}}(u) \varpi(u) \mathrm{d} u, \\
(I I I) \quad & \int_{-\infty}^{\infty} \partial_{u}\left[\phi_{k}(u)\right] \phi_{k^{\prime}}(u) \varpi(u) \mathrm{d} u, \\
(I V) \quad & \int_{-\infty}^{\infty} F^{\prime}(\gamma) \phi_{l}(\gamma) \phi_{l^{\prime}}(\gamma) \varpi(u) \mathrm{d} u, \\
(V) \quad & \int_{-\infty}^{\infty} F(\gamma) \partial_{\gamma}\left[\phi_{l}(\gamma)\right] \phi_{l^{\prime}}(\gamma) \varpi(\gamma) \mathrm{d} \gamma,
\end{array}
$$

where (I)-(III) have already been derived in the previous section. The important point is that only the integrals (II) and (III) contribute to terms with $k^{\prime} \neq k$ in the system for the coefficients $C_{k, l}$ and these integrals are the same as in the case with $F=0$. Consequently, systems with arbitrary $F(\gamma)$ will not violate the 'closedness' property (B.60), resulting in good estimates for the second-order statistics in such systems from the GrChE approximations. Similar to the system (1.1) discussed in the previous section, this closedness property is violated for any perturbation of the dynamics of $\gamma$ in (B.65b) coupling it to the dynamics of $u$.

Appendix C. Approximate second-order statistics of $u$ through the Gaussian closure approximation. The simple Gaussian moment closure approximation of the statistics associated with (1.1) is carried out in the same fashion as the one developed for a related system in $[2,3]$ and relies on the following:

(i) decomposition the system variables into the mean and fluctuations parts, i.e.,

$$
u=\bar{u}+u^{\prime}, \quad \gamma=\bar{\gamma}+\gamma^{\prime}, \quad \text { such that } \overline{u^{\prime}}=\overline{\gamma^{\prime}}=0, \overline{\bar{u}}=\bar{u}, \overline{\bar{\gamma}}=\bar{\gamma},
$$

where the overbar denotes the ensemble average,

(ii) the Ito formula,

(iii) neglecting the third and higher order moments in the evolution of the statistics.

In the case of system (1.1), this procedure leads to the following system with quadratic nonlinearities:

$$
\begin{aligned}
& \frac{\mathrm{d}}{\mathrm{d} t} \bar{u}=-\left(\hat{\gamma}+a_{u} \bar{\gamma}\right) \bar{u}-a_{u} \overline{u^{\prime} \gamma^{\prime}}+f(t), \\
& \frac{\mathrm{d}}{\mathrm{d} t} \bar{\gamma}=-\left(d_{\gamma}+a_{\gamma} \bar{u}\right) \bar{\gamma}-a_{\gamma} \overline{\gamma^{\prime} u^{\prime}},
\end{aligned}
$$




$$
\begin{aligned}
& \frac{\mathrm{d}}{\mathrm{d} t} \overline{u^{\prime 2}}=-2\left(\hat{\gamma}+a_{u} \bar{\gamma}\right) \overline{u^{\prime 2}}-2 a_{u} \bar{u} \overline{u^{\prime} \gamma^{\prime}}+\sigma_{u}^{2}, \\
& \frac{\mathrm{d}}{\mathrm{d} t} \overline{\gamma^{\prime 2}}=-2\left(d_{\gamma}+a_{\gamma} \bar{u}\right) \overline{{\gamma^{\prime}}^{2}}-2 a_{\gamma} \bar{\gamma} \overline{u^{\prime} \gamma^{\prime}}+\sigma_{\gamma}^{2}, \\
& \frac{\mathrm{d}}{\mathrm{d} t} \overline{u^{\prime} \gamma^{\prime}}=-\left(\hat{\gamma}+d_{\gamma}+a_{u} \bar{\gamma}+a_{\gamma} \bar{u}\right) \overline{u^{\prime} \gamma^{\prime}}-a_{u} \bar{u} \overline{\gamma^{\prime 2}}-a_{\gamma} \bar{\gamma} \overline{u^{\prime 2}}
\end{aligned}
$$

which has to be solved in order to obtain the time-dependent statistics of $u$ and $\gamma$.

Appendix D. Analytical expression for the invariant measure for test model (1.1) and its moments in the while noise limit of damping fluctuations. Here, we consider the dynamics of $u(t)$ in (1.1) with $a_{u}=1, a_{\gamma}=0$ in the limit when the random fluctuations $\gamma(t)$ about the mean damping $\hat{\gamma}(t)$ decorrelate very fast. We formally consider this scenario as the white noise limit of the fluctuations in the damping and derive an equilibrium PDF in the case when the mean damping $\hat{\gamma}$ and forcing $f$ are constant; as shown below, the respective PDF is given by

$$
p_{e q}(u)=\frac{N_{0}}{\left(\tilde{\sigma}_{\gamma}^{2} u^{2}+\sigma_{u}^{2}\right)^{\delta_{1}}} \exp \left(\frac{2 f}{\tilde{\sigma}_{\gamma} \sigma_{u}} \arctan \left(\frac{\tilde{\sigma}_{\gamma}}{\sigma_{u}} u\right)\right)
$$

with $\delta_{1}=1+\left(\hat{\gamma}-\frac{1}{2} \tilde{\sigma}_{\gamma}^{2}\right) / \tilde{\sigma}_{\gamma}^{2}$ and the finite constant $\tilde{\sigma}_{\gamma}$ defined below. For timedependent mean damping and/or forcing the moments of the time-dependent PDF can be derived by a successive application of the Itô formula to obtain the evolution of $u^{n}(t)$ in the white noise limit of the damping fluctuations $\gamma(t)$ (see (D.11)). It can be easily checked that in such a case the $n$-th moment of the density $p(u, t)$ is given by

$$
\left\langle u^{n}(t)\right\rangle=\left\langle u^{n}(t)\right\rangle e^{J_{n}\left(t_{0}, t\right)}+\int_{t_{0}}^{t} e^{J_{n}(s, t)}\left(n f(s)\left\langle u^{n-1}(s)\right\rangle+\frac{1}{2} n(n-1) \sigma_{u}^{2}\left\langle u^{n-2}(s)\right\rangle\right) \mathrm{d} s,
$$

where

$$
J_{n}(s, t)=-n \int_{s}^{t} \hat{\gamma}\left(t^{\prime}\right) \mathrm{d} t^{\prime}+\frac{1}{2} n^{2} \tilde{\sigma}_{\gamma}^{2}(t-s)
$$

and we additionally set $\left\langle u(t)^{n-k}\right\rangle=1$ for $n=k$, and $\left\langle u(t)^{n-k}\right\rangle=0$ for $n-k<0, k=1,2$. For time periodic mean damping $\hat{\gamma}(t)=\hat{\gamma}_{0}+\tilde{\gamma}(t)$, with the mean $\hat{\gamma}_{0}$, the exponent (D.3) can be written as

$$
J_{n}(s, t)=\left(-n \hat{\gamma}_{0}+\frac{1}{2} n^{2} \tilde{\sigma}_{\gamma}^{2}\right)(t-s)-n \int_{s}^{t} \tilde{\gamma}\left(t^{\prime}\right) \mathrm{d} t .
$$

The last term in (D.4) is bounded due to the assumed time-periodicity of $\hat{\gamma}$. Elementary estimates show that for bounded forcing $f(t)$ and

$$
-n \hat{\gamma}_{0}+\frac{1}{2} n^{2} \tilde{\sigma}_{\gamma}^{2}<0,
$$

the moments of $p(u, t)$ of order up to $n$ are finite.

The procedure for obtaining the limiting dynamics is as follows. We consider the limit $d_{\gamma} \rightarrow \infty$ to ensure that that the decorrelation time vanishes in the limit. In the 
statistically steady state with respect to the noise $\dot{W}_{\gamma}$ the autocorrelation function of $\gamma(t)$ becomes

$$
\operatorname{Corr}_{\gamma}(\tau)=\langle\gamma(t+\tau) \gamma(t)\rangle_{e q}=\frac{\sigma_{\gamma}^{2}}{2 d_{\gamma}} e^{-d_{\gamma} \tau}
$$

where $\langle\cdot\rangle_{e q}$ denotes the equilibrium ensemble average. Thus, if we keep the following ratio fixed and satisfying

$$
0<\tilde{\sigma}_{\gamma}=\frac{\sigma_{\gamma}}{d_{\gamma}}=\text { const. }
$$

the absolute value of the autocorrelation function (D.6) formally approaches a delta function, i.e.

$$
\lim _{\substack{d_{\gamma} \rightarrow \infty \\ \sigma_{\gamma} / d_{\gamma}=\text { const }}}\left|\operatorname{Corr}_{\gamma}(\tau)\right|=\frac{\tilde{\sigma}}{2} \delta_{0}(\tau),
$$

or equivalently the process $\gamma(t)$ approaches the white noise so that

$$
\mathrm{d} \gamma(t) \rightarrow \tilde{\sigma}_{\gamma} \mathrm{d} W_{\gamma}(t)
$$

As usual in physics and engineering, the white noise limit of colored noise leads to the following Stratonovich SDE (e.g. Gardiner, 1997):

$$
\mathrm{d} u(t)=[-\hat{\gamma}(t) u(t)+f(t)] \mathrm{d} t-\tilde{\sigma}_{\gamma} u(t) \circ \mathrm{d} W_{\gamma}(t)+\sigma_{u} \mathrm{~d} W_{u},
$$

as a consequence of the requirement of non-vanishing correlations $\langle u(t) \gamma(t)\rangle$ before and after the limit. The above white noise limit of (1.1) can be written in Ito form as

$$
\mathrm{d} u(t)=[-\alpha(t) u(t)+f(t)] \mathrm{d} t-\tilde{\sigma}_{\gamma} u(t) \mathrm{d} W_{\gamma}(t)+\sigma_{u} \mathrm{~d} W_{u},
$$

where $\alpha(t)=\hat{\gamma}(t)-\frac{1}{2} \tilde{\sigma}_{\gamma}^{2}$.

D.1. Invariant measure. Here, we determine the stationary solutions of the Fokker-Planck equation associated with (D.11) when $\hat{\gamma}$ and $f$ are constant, i.e.,

$$
\partial_{t} p(u, t)=-\partial_{u}[(-\alpha u+f) p(u, t)]+\frac{1}{2} \partial_{u}^{2}\left[\left(\tilde{\sigma}_{\gamma}^{2} u^{2}+\sigma_{u}^{2}\right) p(u, t)\right] .
$$

The stationary solutions, $p_{e q}(u)$, of (D.12) correspond to invariant measures for $u$ and satisfy

$$
\partial_{u}\left[\left(\tilde{\sigma}_{\gamma}^{2} u^{2}+\sigma_{u}^{2}\right) p_{e q}(u)\right]=2(-\alpha u+f) p_{e q}(u),
$$

which can be easily integrated as

$$
p_{e q}(u)=\frac{N_{0}}{A(u)} \exp \left(\int_{u_{0}}^{u} B\left(u^{\prime}\right) / A\left(u^{\prime}\right) \mathrm{d} u^{\prime}\right)
$$

where

$$
A(u)=\tilde{\sigma}_{\gamma}^{2} u^{2}+\sigma_{u}^{2}, \quad B(u)=2(-\alpha u+f)
$$


The exact solution of (D.14) is easily found by evaluating elementary integrals to be given by a density with fat algebraic tails

$$
p_{e q}(u)=\frac{N_{0}}{\left(\tilde{\sigma}_{\gamma}^{2} u^{2}+\sigma_{u}^{2}\right)^{\delta_{1}}} \exp \left(\frac{2 f}{\tilde{\sigma}_{\gamma} \sigma_{u}} \arctan \left(\frac{\tilde{\sigma}_{\gamma}}{\sigma_{u}} u\right)\right),
$$

with $\delta_{1}=1+\left(\hat{\gamma}-\frac{1}{2} \tilde{\sigma}_{\gamma}^{2}\right) / \tilde{\sigma}_{\gamma}^{2}$, as claimed at the beginning of this section.

Appendix E. Statistics of the system (1.1) with simple parametric uncertainty. Here, instead of white noise driven damping fluctuations in (1.1), we consider the case when the damping fluctuations are given by $\gamma=\sigma_{\gamma} \xi$ (see Case 1 of Section 3.1) so that the path-wise solutions of $u$ are

$$
u(t)=u_{0} e^{-\left(\hat{\gamma}+\sigma_{\gamma} \xi\right)\left(t-t_{0}\right)}+\int_{t_{0}}^{t} e^{-\left(\hat{\gamma}+\sigma_{\gamma} \xi\right)(t-s)} f(s) \mathrm{d} s .
$$

The mean $\langle u\rangle$ is easily found as

$$
\langle u(t)\rangle=\left\langle u_{0}\right\rangle e^{-\hat{\gamma}\left(t-t_{0}\right)+\frac{1}{2} \sigma_{\gamma}^{2}\left(t-t_{0}\right)^{2}}+\int_{t_{0}}^{t} e^{-\hat{\gamma}(t-s)+\frac{1}{2} \sigma_{\gamma}^{2}(t-s)^{2}} f(s) \mathrm{d} s,
$$

and the variance is given by

$$
\begin{aligned}
\operatorname{Var}(u)= & e^{-2 \hat{\gamma}\left(t-t_{0}\right)+2 \sigma_{\gamma}^{2}\left(t-t_{0}\right)^{2}} \operatorname{Var}\left(u_{0}\right) \\
& +2\left\langle u_{0}\right\rangle \int_{t_{0}}^{t} e^{-\hat{\gamma}\left(2 t-t_{0}-s\right)+\frac{1}{2} \sigma_{\gamma}^{2}\left(2 t-t_{0}-s\right)^{2}}\left(1-e^{-\sigma_{\gamma}^{2}\left(t-t_{0}\right)(t-s)}\right) f(s) \mathrm{d} s \\
& +\int_{t_{0}}^{t} \int_{t_{0}}^{t} e^{-\hat{\gamma}\left(2 t-s-s^{\prime}\right)+\frac{1}{2} \sigma_{\gamma}^{2}\left(2 t-s-s^{\prime}\right)^{2}}\left(1-e^{-\sigma_{\gamma}^{2}(t-s)\left(t-s^{\prime}\right)}\right) f(s) f\left(s^{\prime}\right) \mathrm{d} s \mathrm{~d} s^{\prime} .
\end{aligned}
$$

Important facts:

- The mean (E.2) and variance (E.3) are unbounded for $t \in \mathbb{R}$ (due to the quadratic term $\frac{1}{2} \sigma_{\gamma}^{2}\left(t-t_{0}\right)^{2}$ in the respective exponents).

- There exists a metastable phase for both the mean and variance within a time interval $\left[t_{0} t\right]$ satisfying

$$
-\hat{\gamma}\left(t-t_{0}\right)+\sigma_{\gamma}^{2}\left(t-t_{0}\right)^{2}<0 .
$$

- There exists a metastable phase for the mean within a time interval $\left[t_{0} t\right]$ satisfying

$$
-\hat{\gamma}\left(t-t_{0}\right)+\frac{1}{2} \sigma_{\gamma}^{2}\left(t-t_{0}\right)^{2}<0
$$

\section{REFERENCES}

[1] A. Alexanderian, O.P. Le Maitre, H.N. Najm, M. Iskandarani, and O.M. Knio, Multiscale stochastic preconditioners in Non-intrusive spectral projection, J. Sci. Comput., doi:10.1007/s10915-011-9486-2, 1-35, 2011.

[2] M. Branicki, B. Gershgorin, and A.J. Majda, Filtering skill for turbulent signals for a suite of nonlinear and linear Kalman filters, J. Comput. Phys., 231, 1462-1498, 2012. 


\section{LIMITATIONS OF POLYNOMIAL CHAOS FOR UNCERTAINTY QUANTIFICATION}

[3] M. Branicki and A.J. Majda, Quantifying uncertainty for long range forecasting scenarios with model errors in non-Guassian models with intermittency, Nonlin., 25, 2543, doi:10.1088/0951-7715/25/9/2543, 2012

[4] R.H. Cameron and W.T. Martin, The orthogonal development of non-linear functionals in series of Fourier-Hermite functionals, Ann. Math., 48, 385-392, 1947.

[5] A.J. Chorin, Gaussian fields and random flow, J. Fluid Mech., 63, 21-32, 1973.

[6] S.C. Crow and G.H. Canavan, Relationship between a Wiener-Hermite expansion and an energy cascade, J. Fluid Mech., 41, 387-403, 1970.

[7] T. DelSole, Stochastic models of quasigeostrophic turbulence, Surv. Geophys., 25(2), 107-149, 2004.

[8] P. Frauenfelder, C. Schwab, and R.A. Todor, Finite elements for elliptic problems with stochastic coefficients, Comput. Methods Appl. Mech. Eng., 194, 205-228, 2005.

[9] B. Gershgorin, J. Harlim, and A.J. Majda, Test models for improving filtering with model errors through stochastic parameter estimation, J. Comput. Phys., 229(1), 1-31, 2010.

[10] B. Gershgorin, J. Harlim, and A.J. Majda, Improving filtering and prediction of spatially extended turbulent systems with model errors through stochastic parameter estimation, J. Comput. Phys., 229(1), 32-57, 2010.

[11] R.G. Ghanem, and P.D. Spanos, Stochastic Finite Elements: A Spectral Approach, SpringerVerlag, 1991.

[12] T. Hou, W. Luo, B. Rozovskii, and H-M. Zhou, Wiener Chaos expansions and numerical solutions of randomly forced equations of fluid mechanics, J. Comput. Phys., 216, 687706, 2006.

[13] O.P. Le Maitre, O.M. Knio, H.N. Najm, and R.G. Ghanem, A stochastic projection method for fluid flow I. Basic formulation, J. Comput. Phys., 173, 481-511, 2001.

[14] K. Knopp, Theory and Application of Infinite Series, Dover Publications, 1990.

[15] O.P. Le Maitre, O.M. Knio, H.N. Najm, and R.G. Ghanem, Uncertainty propagation using Wiener-Haar expansions, J. Comput. Phys., 197(1), 28-57, 2004.

[16] O.P. Le Maitre, H.N. Najm, R.G. Ghanem, and O.M. Knio, Multi-resolution analysis of Wiener-type un- certainty propagation schemes, J. Comput. Phys., 197(2), 502-531, 2004.

[17] O.P. Le Maitre, H.N. Najm, P.P. Pebay, R.G. Ghanem, and O.M. Knio, Multi-resolutionanalysis scheme for uncertainty quantification in chemical systems, SIAM J. Sci. Comput., 29(2), 864-889, 2007.

[18] R.S. Liptser and A.N. Shiryaev, Statistics of Random Process, Springer-Verlag, 2nd ed., New York, 2001.

[19] W. Luo, Wiener Chaos Expansion and Numerical Solutions of Stochastic Partial Differential Equations, PhD Thesis, Caltech, 2006.

[20] W. Luo, Wiener Chaos Expansion and Numerical Solutions of Stochastic PDE, VDM Verlag, 2010.

[21] O.P. Le Maitre, L. Mathelin, O.M. Knio, and M.Y. Hussaini, Asynchronous time integration for polynomial chaos expansion of uncertain periodic dynamics, Disc. Cont. Dyn. Sys., 28(1), 199-226, 2010.

[22] A.J. Majda and M. Branicki, Lessons in UQ for Complex Turbulent Systems, Disc. Cont. Dyn. Sys., 2(9), 3133-3221, doi:10.3934/dcds.2012.32.3133, 2012

[23] L. Mathelin and O.P. Le Maitre, Uncertainty quantification in a chemical system using error estimated-based adaptation, Theor. Comput. Fluid Dyn., doi:10.1007/s00162-010-0210-x, 2010.

[24] H.G. Matthies and A. Keese, Galerkin methods for linear and nonlinear elliptic stochastic partial differential equations, Comput. Methods Appl. Mech. Eng., 194, 1295-331, 2005.

[25] W.C. Meecham and A. Siegel, Wiener-Hermite expansion in model turbulence at large Reynolds number, Phys. Fluids, 7, 1178-1190, 1964.

[26] W.C. Meecham and A. Siegel, Use of the Wiener-Hermite expansion for nearly normal turbulence, J. Fluid Mech., 32, 225-249, 1968.

[27] R. Mikulevicius and B. Rozovskii, Stochastic Navier-Stokes equations for turbulence flow, SIAM J. Math. Anal., 35, 1250-1310, 2004.

[28] H.N. Najm, B. Debusschere, Y. Marzouk, S. Widmer, and O.P. Le Maitre, Uncertainty quantification in chemical systems, Int. J. Num. Eng., 80(6), 789-814, 2009.

[29] C.L. Pettit and P.S. Beran, Spectral and multiresolution Wiener expansions of oscillatory stochastic processes, J. Sound Vib., 294, 752-779, 2006.

[30] C. Prévot and M. Röckner, A concise Course on Stochastic Partial Differential Equations, Springer, 2000.

[31] S. Sakamoto and R. Ghanem, Polynomial chaos decomposition for the simulation of nonGaussian non-stationary stochastic processes, J. Eng. Mech., 128, 190-201, 2002. 
[32] Y. Ogura, A consequence of the zero-fourth-cumulant approximation in the decay of isotropic turbulence, J. Fluid Mech., 16, 33-40, 1963.

[33] B. Oksendal, Stochastic Differential Equations, Springer, 5ed., 2010.

[34] S.A. Orszag and L.R. Bissonnette, Dynamical properties of truncated Wiener-Hermite expansions, Phys. Fluids, 10, 2603-2613, 1967.

[35] C. Schwab and R.A. Todor, Sparse finite elements for elliptic problems with stochastic data, Numer. Math., 95, 707-734, 2003.

[36] X. Wan and G.E. Karniadakis, An adaptive multi-element generalized polynomial chaos method for stochastic differential equations, J. Comput. Phys., 209, 617-642, 2005.

[37] X. Wan and G.E. Karniadakis, Multi-element generalized polynomial chaos for arbitrary probability measures, SIAM J. Sci. Comput., 28(3), 901-928, 2006.

[38] N. Wiener, The homogeneous chaos, Am. J. Math., 60, 897-936, 1938.

[39] N. Wiener, The use of statistical theory in the study of turbulence, Proc. Fifth Int. Cong. Appl. Mech., New York: Wiley, 356, 1939.

[40] D. Xiu and G. E. Karniadakis, Modeling uncertainty in steady state diffusion problems via generalized polynomial chaos, Comput. Methods Appl. Mech. Engin., 191(43), 4927-4948, 2002.

[41] D. Xiu and G.E. Karnidakis, The Wiener-Askey polynomial chaos for stochastic differential equations, SIAM J. Sci. Comput., 24, 619-644, 2002.

[42] D. Xiu, D. Lucor, C.H. Su, and G.E. Karniadakis, Stochastic modeling of flow-structure interactions using generalized polynomial chaos, J. Fluid Engin., 124, 51-59, 2002.

[43] D. Xiu and G.E. Karnidakis, Modeling uncertainty in flow simulations via generalized polynomial chaos, J. Comput. Phys., 187, 137-167, 2003.

[44] D. Xiu and J.S. Hesthaven, High-order collocation methods for differential equations with random inputs, SIAM J. Sci. Comput., 27(3), 1118-1139, 2005.

[45] D. Zhang and Z. Lu, An efficient, high-order perturbation approach for flow in random porous media via KarhunenLoéve and polynomial expansion, J. Comput. Phys., 194, 773-794, 2004. 